\title{
A one Field Full Discontinuous Galerkin Method for Kirchhoff-Love Shells Applied to Fracture Mechanics
}

\author{
G. Becker ${ }^{\mathrm{a}, 1}$, C. Geuzaine ${ }^{\mathrm{b}}$, L. Noels ${ }^{\mathrm{a}, *}$ \\ ${ }^{a}$ University of Liège, \\ Department of Aerospace and Mechanical Engineering Department, \\ Computational \&s Multiscale Mechanics of Materials, \\ Chemin des Chevreuils 1, B-4000 Liège, Belgium \\ ${ }^{b}$ University of Liége, \\ Department of Electrical Engineering and Computer Science \\ B28 P32, B-4000 Liège, Belgium
}

\begin{abstract}
In order to model fracture, the cohesive zone method can be coupled in a very efficient way with the Finite Element method. Nevertheless, there are some drawbacks with the classical insertion of cohesive elements. It is well known that, on one the hand, if these elements are present before fracture there is a modification of the structure stiffness, and that, on the other hand, their insertion during the simulation requires very complex implementation, especially with parallel codes. These drawbacks can be avoided by combining the cohesive method with the use of a discontinuous Galerkin formulation. In such a formulation, all the elements are discontinuous and the continuity is weakly ensured in a stable and consistent way by inserting extra terms on the boundary of elements. The recourse to interface elements allows to substitute them by cohesive elements at the onset of fracture.

The purpose of this paper is to develop this formulation for Kirchhoff-Love plates and shells. It is achieved by the establishment of a full DG formulation of shell combined with a cohesive model, which is adapted to the special thickness discretization of shell formulation. In fact, this cohesive model is applied on resulting reduced stresses which are the basis of thin structures formulations. Finally, numerical examples demonstrate the efficiency of the method.

Keywords: Discontinuous Galerkin method, shells, Kirchhoff-Love, finite-elements, fracture mechanics, cohesive element

* Corresponding author

Email address: L.Noels@ulg.ac.be (L. Noels)

${ }^{1} \mathrm{PhD}$ candidate at the Belgian National Fund for Education at the Research in Industry and Farming.
\end{abstract}




\section{Introduction}

When designing thin structures, tearing prediction remains a challenging modeling task. This increases the interest of fracture and rupture numerical models for thin bodies, which must be able to take into account a through-thethickness fracture.

Among the different approaches in fracture mechanics, the cohesive zone concept pioneered by Dugdale [1] and Barenblatt [2] is based on a "Traction Separation Law" (TSL), which gives a relationship between the tension and the opening of the crack faces $(\Delta)$, and can be easily combined with Finite Element (FE) methods [3-10]. In such an approach, cohesive elements, integrating the TSL, are inserted as interface elements between bulk elements. Unfortunately, as it is extensively discussed in [11, 12], the two classical methods considered to introduce the cohesive elements suffer from severe limitations. On the one hand, an intrinsic cohesive law $[4,6,9,13]$, for which cohesive elements are introduced at the beginning of the simulation, has to consider the pre-fracture stage by inserting an initial slope in the TSL (see Fig. 1). This initial slope must tend to infinity to ensure a correct wave propagation in the structure, which leads to some numerical problems [14]. On the other hand, an extrinsic cohesive law, where the cohesive elements are inserted on the fly during the simulation when a fracture criterion is reached $[3,7,8,10]$, requires a very complex implementation [15-17] due to the inherent difficulty associated with propagating topological changes in the mesh. As a large number of degree of freedoms (dofs) is needed to obtain a convergence in a fracture problem [7], a parallel implementation can be required to perform large simulations in an admissible computational time, further complicating the implementation.

Some alternatives have been suggested [18-21] to compensate these different limitations, and one promising method, especially when considering 3D parallel simulations, is a new approach based on the combination of a full discontinuous Galerkin (DG) formulation and an extrinsic cohesive law. This method was pioneered by J. Mergheim et al. [22], by R. Radovitzky et al. [11, 12], and by Prechtel et al. [23]. In this method, interface elements are inserted at the beginning of the simulation between discontinuous bulk elements to weakly ensure the compatibility condition in a stable and consistent manner. When a fracture criterion is reached, this interface element is then very easily replaced by a cohesive element. This method has recently been implemented for 3D elements by R. Radovitzky et al. in [12], where it was shown that this approach is scalable when parallelized and does not require the use of complex topological information. Moreover, this method can be implemented easily into existing FE codes. For completeness, let us note that the combination of cohesive law and discontinuous Galerkin method was also achieved by using a space-time discontinuous method [21].

This method can be seen as complementary to the XFEM method [24-29], among others. The XFEM formulation, pioneered by Moës and Belytschko [26] allows to take into account discontinuities inside elements, and is thus very appealing to model crack propagation. The most common XFEM approaches 
enrich nodes with the linear elastic fracture mechanics solution in order to extract the stress intensity factors, and to propagate a crack using the maximum hoop criterion. In the method based on the DG/extrinsic law combination, cracks can be initiated, merged, or propagate, without implementing explicitly crack propagation criteria, as the solution results naturally from the minimization of energy. Moreover as the DG/extrinsic law combination is well suited for dynamics and large computations in parallel, it makes a good alternative to XFEM for problems of fragmentation as for structures subjected to shock, blast... Although the DG/extrinsic law combination suffers from having cracks forced to follow the elements edges, and thus requires a fine mesh to capture the solution, as recently demonstrated by Duflot et al. [29] for 3D problems, the XFEM method also requires fine mesh around the crack tip to capture the crack path. On the implementation point of view, the DG/extrinsic law combination can easily be introduced in an existing parallel code, while the implementation of XFEM requires more attention. Indeed implementation of the XFEM method is challenging when the crack path is near a node, which requires re-meshing [29], when applying Dirichlet boundary conditions... Also, Parrallelizing the XFEM is not straightforward, which makes the DG/extrinsic law combination a good candidate to solve large scale problems.

In a recent paper, the authors extended the combination of a full discontinuous Galerkin (DG) formulation and an extrinsic cohesive law to Euler Bernoulli beams [30], and the present work wants to develop this formulation for KirchhoffLove shells fracture problems. Toward this end, a new one-field full discontinuous Galerkin (full-DG) discretization of the shell equations is obtained from the extension of the work of several authors [31-36] who have developed a $C^{0} / \mathrm{DG}$ formulation for thin bodies. In these works a DG method is used to weakly enforce the $C^{1}$ continuity required by high-order formulations of beams, plates and shells, which leads to displacement-field only methods (nodes have no degree of freedom of rotation). However to extend such a formulation to fracture, it is convenient to consider discontinuous test functions in order to insert interface elements. The study of the new resulting full-DG formulation shows that this formulation is consistent, stable (if stabilization parameters are larger than a mesh-independent constant) and that it converges in the $\mathbf{L}^{2}$ norm with the optimum rate.

In order to enhance this full DG formulation with fracture mechanics, the interface element should integrate the TSL upon the onset of fracture. However, as it is discussed in [30], the model of a "through the thickness fracture" is not straightforward for thin bodies since the thickness is modeled implicitly in the mesh discretization. To avoid the evaluation of the TSL at different points on the thickness, authors suggested in [30] to apply the cohesive principles directly to the resultant stresses (bending and membrane) in terms of the resultant openings (angle and mid-plane openings). The new traction separation law is then defined in such a way that the model respects the energetic balance during the fracture process for any coupled bending-traction loadings. This model is extended here to linear shell elements by considering a combination of the different fracture modes. 
The article is organized as follows. First, the governing equations of continuum mechanics of shells are recalled in section 2. Afterward, in section 3, these equations are formulated within a full-DG framework and the numerical properties are then, on the one hand, studied in an analytical way, and, on the other hand illustrated by a numerical example. In section 4 , an original cohesive zone model based on the resultant stresses is presented and is coupled with the full-DG formulation in order to take into account brittle fracture. The next section deals with some considerations about the implementation of the method. Section 6 presents several numerical applications of fracture testing to demonstrate the ability of the presented framework to simulate fracture problems of thin bodies. Finally some concluding remarks are drawn.

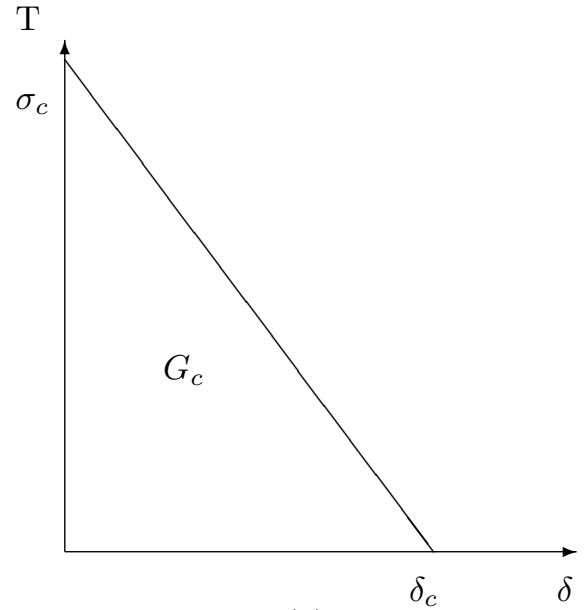

(a)

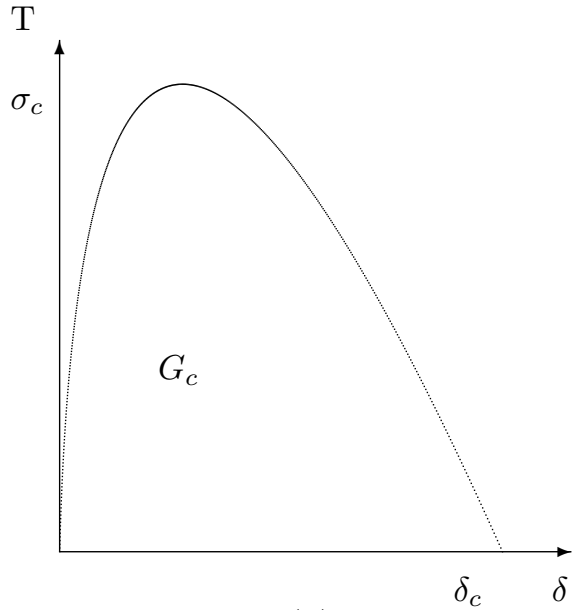

(b)

Figure 1: Linearly decreasing monotonic (a) extrinsic and (b) intrinsic "Traction Separation Law".

\section{Continuum mechanics of thin bodies}

The continuum mechanics of thin structures is well established and can be found in several references $[34,35,37,38]$. For this reason, this section presents only the important results and the notations required to develop the full DG theory. More details can be found in the cited papers, and in particular the last two references use exactly the same notations and conventions as in this paper.

\subsection{Kinematics of the shell}

A thin body can be described by considering its mid-surface section as a Cosserat plane $\mathcal{A}$ and a third coordinate, representing the thickness, belonging 


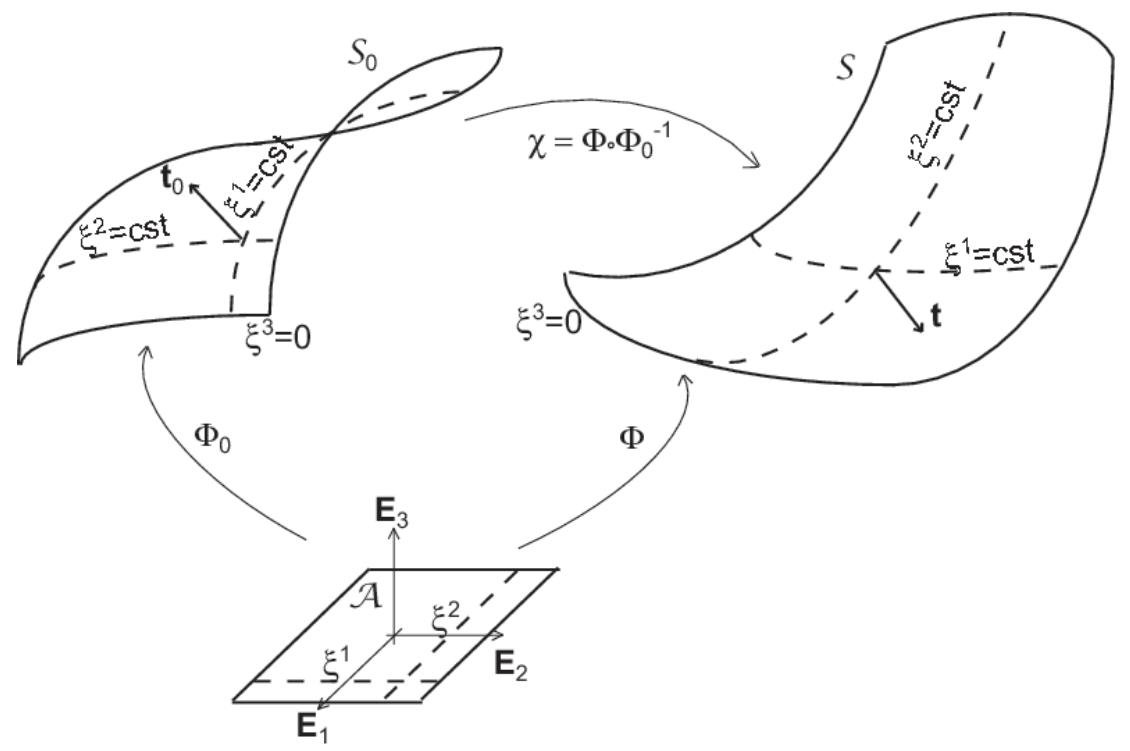

Figure 2: Description of the different configurations of the shell.

to the interval $\left[h_{\min } ; h_{\max }\right]$. In the reference frame $\boldsymbol{E}_{I}$, this representation is written $\boldsymbol{\xi}=\sum_{I=1}^{3} \xi^{I} \boldsymbol{E}_{I}: \mathcal{A} \times\left[h_{\min } ; h_{\max }\right] \rightarrow \mathbb{R}^{3}$. Hereinafter, a subscript will be used to refer to values expressed in the considered basis, while a superscript will be used to refer to values expressed in the conjugate basis. Of course, for the initial frame, $\boldsymbol{E}_{I}=\boldsymbol{E}^{I}$. Roman letters as a subscript or superscript substitute for integers between one and three, while Greek letters substitute for integers one or two. The representation of the body in the inertial frame is illustrated in Fig. 2. using $\varphi\left(\xi^{1}, \xi^{2}\right): \mathcal{A} \rightarrow \mathbb{R}^{3}$ the mapping of the mid-surface and $\boldsymbol{t}: \mathcal{A} \rightarrow \mathrm{S}^{2}=\left\{\left.\boldsymbol{t} \in \mathbb{R}^{3}\right|_{\|\boldsymbol{t}\|=1}\right\}$ the director of the mid-surface, with $\mathrm{S}^{2}$ the unit sphere manifold. A configuration $\mathcal{S}$ of the shell is represented by the manifold of position $\boldsymbol{x}$, which is obtained by the mapping $\boldsymbol{\Phi}: \mathcal{A} \times\left[h_{\min } ; h_{\max }\right] \rightarrow \mathcal{S}$,

$$
\boldsymbol{x}=\boldsymbol{\Phi}\left(\xi^{I}\right)=\boldsymbol{\varphi}\left(\xi^{\alpha}\right)+\xi^{3} \boldsymbol{t}\left(\xi^{\alpha}\right) .
$$

By convention, $\mathcal{S}$ refers to the current configuration of the shell, while the reference configuration $\mathcal{S}_{0}$ is obtained by the mapping $\boldsymbol{\Phi}_{0}$.

\subsection{Governing equations of the linear shell}

The governing equations of a thin body are obtained by integrating on the thickness the equations of force and moment equilibrium, leading to

$$
\begin{aligned}
& \bar{\rho} \ddot{\boldsymbol{\varphi}}-\frac{1}{\bar{j}}\left(\bar{j} \boldsymbol{n}^{\alpha}\right)_{, \alpha}=0 \quad \text { on } \mathcal{A}, \text { and } \\
& \frac{1}{\bar{j}}\left(\bar{j} \tilde{\boldsymbol{m}}^{\alpha}\right)_{, \alpha}-\boldsymbol{l}+\lambda \boldsymbol{t}=0 \quad \text { on } \mathcal{A},
\end{aligned}
$$


where $\lambda$ is an undefined pressure, where $\bar{\rho}=h \rho$ is the density by unit of surface with $h$ the thickness and where $\bar{j}=\left\|\boldsymbol{\varphi}_{, 1} \wedge \boldsymbol{\varphi}_{, 2}\right\|$. Furthermore, the integration on the thickness of the Cauchy stress tensor $\boldsymbol{\sigma}[37,38]$ leads to the definition of

$$
\begin{aligned}
\boldsymbol{n}^{\alpha} & =\frac{1}{\bar{j}} \int_{h_{\min }}^{h_{\max }} \boldsymbol{\sigma} \boldsymbol{g}^{\alpha} \operatorname{det}(\boldsymbol{\nabla} \boldsymbol{\Phi}) d \xi^{3}, \\
\boldsymbol{m}^{\alpha} & =\frac{1}{\bar{j}} \boldsymbol{t} \wedge \int_{h_{\min }}^{h_{\max }} \xi^{3} \boldsymbol{\sigma} \boldsymbol{g}^{\alpha} \operatorname{det}(\boldsymbol{\nabla} \boldsymbol{\Phi}) d \xi^{3}=\boldsymbol{t} \wedge \tilde{\boldsymbol{m}}^{\alpha}, \text { and } \\
\boldsymbol{l} & =\frac{1}{\bar{j}} \int_{h_{\min }}^{h_{\max }} \boldsymbol{\sigma} \boldsymbol{g}^{3} \operatorname{det}(\boldsymbol{\nabla} \boldsymbol{\Phi}) d \xi^{3},
\end{aligned}
$$

respectively the resultant stress vector, the resultant torque vector and the resultant across-the-thickness stress vector. Note that in equations (2) and (3) inertial angular forces are neglected ${ }^{2}$ and the external forces are not considered ${ }^{3}$.

In order to define the stress components, the resultant stress vectors are decomposed in the convected basis, as

$$
\begin{aligned}
\boldsymbol{n}^{\alpha} & =n^{\alpha \beta} \boldsymbol{\varphi}_{0, \beta}+l^{\alpha} \boldsymbol{t}_{0}=\left(\tilde{n}^{\alpha \beta}+\lambda_{\mu}^{\beta} \tilde{m}^{\alpha \mu}\right) \boldsymbol{\varphi}_{0, \beta}+l^{\alpha} \boldsymbol{t}_{0}, \\
\boldsymbol{l} & =l^{\alpha} \boldsymbol{\varphi}_{0, \alpha}, \text { and } \\
\tilde{\boldsymbol{m}}^{\alpha} & =\tilde{m}^{\alpha \beta} \boldsymbol{\varphi}_{0, \beta}+\tilde{m}^{3 \alpha} \boldsymbol{t}_{0} .
\end{aligned}
$$

In these expressions, $\tilde{n}^{\alpha \beta}$ is the membrane stress, $\tilde{m}^{\alpha \beta}$ is the stress couple resultant, $l^{\alpha}$ is the out-of-plane stress resultant and $\tilde{m}^{\alpha 3}$ is the out-of-plane stress couple and $\lambda_{\mu}^{\beta}=\boldsymbol{t}_{0, \mu} \cdot \boldsymbol{\varphi}_{0}^{, \beta}$ characterizes the curvature of the shell. Under Kirchhoff-Love shell assumption, $l^{\alpha}$ can be neglected, but it is kept temporarily in the equations in order to develop the full-DG formulation.

This set of governing equations is accompanied by conventional boundary conditions applied on the boundary $\partial \mathcal{A}$ of the mid-surface $\mathcal{A}$ (see [34] for details).

\subsection{Constitutive behavior}

The constitutive behavior considered in this paper, assumes a linear elastic response between deformation and stress tensors and small displacements $\boldsymbol{u}=$ $\boldsymbol{\varphi}-\boldsymbol{\varphi}_{0}$ of the mid-surface ans small section direction variations $\Delta \boldsymbol{t}=\boldsymbol{t}-\boldsymbol{t}_{0}$ are assumed.

Since integrated stresses have been decomposed into membrane, shearing and bending stresses acting on the mid-surface convected basis, following [38], the deformations are also separated into membrane $\varepsilon$, shearing $\delta$ and torque $\rho$ strain

\footnotetext{
${ }^{2}$ The inertial angular forces can be neglected if the thickness is sufficiently thin which is usually the case for thin bodies formulations.

${ }^{3}$ The interested reader can refer to [34] to develop the formulation with external forces.
} 
components,

$$
\begin{aligned}
\varepsilon_{\alpha \beta} & =\frac{1}{2} \boldsymbol{\varphi}_{, \alpha} \cdot \boldsymbol{\varphi}_{, \beta}-\frac{1}{2} \boldsymbol{\varphi}_{0, \alpha} \cdot \boldsymbol{\varphi}_{0, \beta}=\frac{1}{2} \boldsymbol{\varphi}_{0, \alpha} \cdot \boldsymbol{u}_{, \beta}+\frac{1}{2} \boldsymbol{u}_{, \alpha} \cdot \boldsymbol{\varphi}_{0, \beta} \\
\delta_{\alpha} & =\frac{\boldsymbol{u}, \alpha}{2} \cdot \boldsymbol{t}_{0}+\Delta \boldsymbol{t} \cdot \boldsymbol{\varphi}_{0, \alpha} \\
\rho_{\alpha \beta} & =0, \text { and } \\
& =\boldsymbol{\varphi}_{, \alpha} \cdot \boldsymbol{t}_{, \beta}-\boldsymbol{\varphi}_{0, \alpha} \cdot \boldsymbol{t}_{0, \beta} \\
& \boldsymbol{\varphi}_{0, \alpha \beta} \cdot \boldsymbol{t}_{0} \frac{e_{\mu \eta 3}}{\bar{j}_{0}} \boldsymbol{u}_{, \mu} \cdot\left(\boldsymbol{\varphi}_{0, \eta} \wedge \boldsymbol{t}_{0}\right)+\frac{e_{\mu \eta 3}}{\bar{j}_{0}} \boldsymbol{u}_{, \mu} \cdot\left(\boldsymbol{\varphi}_{0, \alpha \beta} \wedge \boldsymbol{\varphi}_{0, \eta}\right)-\boldsymbol{u}_{, \alpha \beta} \cdot \boldsymbol{t}_{0}
\end{aligned}
$$

to define the elastic constitutive relations between the effective stresses and strains,

$$
\begin{aligned}
& \tilde{n}^{\alpha \beta}=\frac{E\left(h_{\max }-h_{\min }\right)}{1-\nu^{2}} \mathcal{H}^{\alpha \beta \gamma \delta} \varepsilon_{\gamma \delta} \quad=\mathcal{H}_{n}^{\alpha \beta \gamma \delta} \varepsilon_{\gamma \delta}, \\
& \tilde{m}^{\alpha \beta}=\frac{E\left(h_{\max }-h_{\min }\right)^{3}}{12\left(1-\nu^{2}\right)} \mathcal{H}^{\alpha \beta \gamma \delta} \rho_{\gamma \delta}=\mathcal{H}_{m}^{\alpha \beta \gamma \delta} \rho_{\gamma \delta} \text {, and } \\
& l^{\alpha}=G\left(h_{\max }-h_{\min }\right) \frac{A^{\prime}}{A} \mathcal{H}^{\alpha \beta} \gamma_{\beta}=\mathcal{H}_{s}^{\alpha \beta} \gamma_{\beta},
\end{aligned}
$$

where $E$ is the Young modulus, $\nu$ the Poisson's ration, $G$ is the shear modulus, $\gamma=2 \delta$ and where

$$
\begin{aligned}
\mathcal{H}^{\alpha \beta \gamma \delta}= & \nu \boldsymbol{\varphi}_{0}^{\alpha} \cdot \boldsymbol{\varphi}_{0}^{, \beta} \boldsymbol{\varphi}_{0}^{, \gamma} \cdot \boldsymbol{\varphi}_{0}^{, \delta}+\frac{1}{2}(1-\nu) \boldsymbol{\varphi}_{0}^{, \alpha} \cdot \boldsymbol{\varphi}_{0}^{, \gamma} \boldsymbol{\varphi}_{0}^{, \delta} \cdot \boldsymbol{\varphi}_{0}^{, \beta}+ \\
& \frac{1}{2}(1-\nu) \boldsymbol{\varphi}_{0}^{, \alpha} \cdot \boldsymbol{\varphi}_{0}^{, \delta} \boldsymbol{\varphi}_{0}^{, \gamma} \cdot \boldsymbol{\varphi}_{0}^{, \beta} \\
\mathcal{H}^{\alpha \beta}= & \boldsymbol{\varphi}_{0}^{, \alpha} \cdot \boldsymbol{\varphi}_{0}^{, \beta} .
\end{aligned}
$$

with in this last expression $\varphi_{0}^{, \alpha}$ denotes, with an abuse of notation, the conjugate basis to $\varphi_{0, \alpha}$.

Furthermore, although in the Kirchhoff-Love formulation the shearing is neglected leading to $l^{\alpha}=0$, the relation (15) is still considered as it will be required when developing the full discontinuous Galerkin formulation. Indeed, as usual components of shell equations $\boldsymbol{n}^{\alpha}$ and $\tilde{\boldsymbol{m}}^{\alpha}$ do not consider explicitly the normal displacement ${ }^{4}$, equation (15) will allow ensuring that continuity of the normal deflection across interfaces is weakly satisfied.

\section{Full discontinuous Galerkin formulation}

This section suggests a novel framework for numerical approximation of the shell equations, based on a discontinuous polynomial approximation of the unknown field $\boldsymbol{\varphi}$. Indeed, under the Kirchhoff-Love assumption, the unit vector $\boldsymbol{t}$ can be stated in terms of $\boldsymbol{\varphi}$, see [34] for details. In the new formulation, the

\footnotetext{
${ }^{4}$ The normal displacement appears only in $\tilde{\boldsymbol{m}}^{\alpha}$ via its first derivative.
} 
discontinuities of test functions are accounted for using a novel full-DG formulation, contrarily to previous DG formulations for thin bodies [31, 33, 34, 36, 39] where this field was continuous. This discontinuity requirement is a first step toward the application to fracture mechanics, as it will be seen later on.

\subsection{Weak formulation of the problem}

The finite-element discretization is performed at the mid-surface level of the initial configuration of the shell $\mathcal{S}_{0}$. Accordingly, the mid-surface $\mathcal{A}$ is approximated by a discretization $\mathcal{A}_{h}$ into finite-elements $\mathcal{A}_{e}$, with $\mathcal{A} \simeq \mathcal{A}_{h}=\bigcup_{e} \overline{\mathcal{A}}_{e}$. In this last equation, $\overline{\mathcal{A}}_{e}$ is the union of the open domain $\mathcal{A}_{e}$ with its boundary $\partial \mathcal{A}_{e}$. The boundary $\partial \mathcal{A}_{e}$ of an element $\mathcal{A}_{e}$ can be common with the boundary of $\mathcal{A}_{h}$, with

$$
\begin{aligned}
\partial_{U} \mathcal{A}_{e} & =\partial \mathcal{A}_{e} \cap \partial_{U} \mathcal{A}_{h}, \\
\partial_{T} \mathcal{A}_{e} & =\partial \mathcal{A}_{e} \cap \partial_{T} \mathcal{A}_{h}, \\
\partial_{M} \mathcal{A}_{e} & =\partial \mathcal{A}_{e} \cap \partial_{M} \mathcal{A}_{h}, \\
\partial_{N} \mathcal{A}_{e} & =\partial \mathcal{A}_{e} \cap \partial_{N} \mathcal{A}_{h} .
\end{aligned}
$$

The remaining part of the boundary $\partial \mathcal{A}_{e}$ is shared with another finite element and, is thus part of the interior boundary $\partial_{I} \mathcal{A}_{h}$, with

$$
\begin{aligned}
\partial_{I} \mathcal{A}_{e} & =\partial \mathcal{A}_{e} \backslash \partial \mathcal{A}_{h} \\
& =\partial \mathcal{A}_{e} \cap \partial_{I} \mathcal{A}_{h}, \text { with } \partial_{I} \mathcal{A}_{h}=\bigcup_{e} \partial \mathcal{A}_{e} \backslash \partial \mathcal{A}_{h}
\end{aligned}
$$

Instead of seeking the exact solution $\varphi$ of the problem, a polynomial approximation $\varphi_{h} \in \mathrm{U}_{\mathrm{h}}^{k}$ constitutes the solution to the finite element discretization. In this work, a discontinuous polynomial approximation between the elements is considered, and therefore the derivatives of the displacement field are also allowed to be discontinuous on the element boundaries, leading to the definition of the displacement manifold and of its constrained counterpart

$$
\begin{aligned}
\mathrm{U}_{\mathrm{h}}^{k} & =\left\{\left.\left.\boldsymbol{\varphi}_{h} \in \mathbf{H}^{0}\left(\mathcal{A}_{h}\right)\right|_{\varphi_{h}}\right|_{\mathcal{A}_{e} \in \mathbb{P}^{k}} \forall \mathcal{A}_{e} \in \mathcal{A}_{h}\right\} \subset \mathrm{U}^{\mathrm{f}}\left(\mathcal{A}_{h}\right), \\
\mathrm{U}_{\mathrm{h} c}^{k} & =\left\{\left.\left.\delta \boldsymbol{\varphi} \in \mathrm{U}_{\mathrm{h}}^{k}\right|_{\delta \varphi}\right|_{\partial_{U} \mathcal{A}}=0\right\} \subset \mathrm{U}_{c}^{\mathrm{f}}\left(\mathcal{A}_{h}\right),
\end{aligned}
$$

with $\mathrm{U}^{\mathrm{f}}\left(\mathcal{A}_{h}\right)=\prod_{e} \mathbf{H}^{2}\left(\mathcal{A}_{e}\right)$ for polynomial approximations $k>1$ and with $\mathrm{U}_{c}^{\mathrm{f}}\left(\mathcal{A}_{h}\right)=\left\{\left.\delta \boldsymbol{\varphi} \in \mathrm{U}^{\mathrm{f}}\left(\mathcal{A}_{h}\right)\right|_{\left.\delta \varphi\right|_{\partial_{U} \mathcal{A}}=0}\right\}$.

The purpose of this section is to establish a weak form of the problem, stated by the set of Eqs. (2-3) completed with appropriated boundary conditions, for an approximation $\varphi_{h} \in \mathrm{U}_{\mathrm{h}}^{k}$. Owing to the definition of this manifold, see Eq. (20), the displacement field and the test functions are discontinuous across elementinterfaces, which has to be accounted for when establishing the new weak form of the problem. 
Multiplying Eq. (2) by a test function $\delta \varphi \in \mathrm{U}_{\mathrm{h} c}^{k}$, and Eq. (3) by the corresponding variation of unit vector $\delta \Delta \boldsymbol{t}=\Delta \boldsymbol{t}(\delta \boldsymbol{\varphi})$, states the problem as finding $\varphi_{h} \in \mathrm{U}_{\mathrm{h}}^{k}$ such that

$$
\begin{aligned}
\sum_{e} \int_{\mathcal{A}_{e}} \bar{\rho} \ddot{\boldsymbol{\varphi}}_{h} \cdot \delta \boldsymbol{\varphi} d \mathcal{A}= & \sum_{e}\left\{\int_{\mathcal{A}_{e}}\left(\bar{j}_{0} \boldsymbol{n}^{\alpha}\left(\boldsymbol{\varphi}_{h}\right)\right)_{, \alpha} \cdot \delta \boldsymbol{\varphi} d \mathcal{A}\right. \\
& \left.+\int_{\mathcal{A}_{e}}\left[\left(\bar{j}_{0} \tilde{\boldsymbol{m}}^{\alpha}\left(\boldsymbol{\varphi}_{h}\right)\right)_{, \alpha}-\bar{j}_{0} \boldsymbol{l}\right] \cdot \delta \Delta \boldsymbol{t} d \mathcal{A}\right\}
\end{aligned}
$$

$\forall \delta \varphi \in \mathrm{U}_{\mathrm{h} c}^{k}$. In this equation, relation $\boldsymbol{t} \cdot \delta \Delta \boldsymbol{t}=0$ has been considered, as well as a first order approximation of the balance equations. Integration by parts of these integrals followed by the application of the Gauss theorem leads to

$$
\begin{aligned}
\sum_{e} a_{d}^{e}\left(\boldsymbol{\varphi}_{h}, \delta \boldsymbol{\varphi}\right)= & -\sum_{e} a_{n}^{e}\left(\boldsymbol{\varphi}_{h}, \delta \boldsymbol{\varphi}\right)+\sum_{e} \int_{\partial \mathcal{A}_{e}} \bar{j}_{0} \boldsymbol{n}^{\alpha}\left(\boldsymbol{\varphi}_{h}\right) \cdot \delta \boldsymbol{\varphi}_{\alpha} d \mathcal{A} \\
& -\sum_{e} a_{m}^{e}\left(\boldsymbol{\varphi}_{h}, \delta \boldsymbol{\varphi}\right)+\sum_{e} \int_{\partial \mathcal{A}_{e}} \bar{j}_{0} \tilde{\boldsymbol{m}}^{\alpha}\left(\boldsymbol{\varphi}_{h}\right) \cdot \delta \Delta \boldsymbol{t} \nu_{\alpha} d \mathcal{A} \\
& +\sum_{e} a_{s}^{e}\left(\boldsymbol{\varphi}_{h}, \delta \boldsymbol{\varphi}\right)-\sum_{e} \int_{\partial \mathcal{A}_{e}} \bar{j}_{0} \boldsymbol{l} \cdot \int_{\alpha} \delta \Delta \boldsymbol{t}_{\beta} \boldsymbol{\varphi}_{0}^{, \beta} d \alpha^{\prime} \nu_{\alpha} d \mathcal{A}
\end{aligned}
$$

with $\delta \Delta \boldsymbol{t}=\delta \Delta \boldsymbol{t}_{\beta} \boldsymbol{\varphi}_{0}^{, \beta}$, with an unusual integration by parts on $\delta \Delta \boldsymbol{t}$ in place of $\bar{j}_{0} \boldsymbol{l}$ for the third term, and with the following bi-linear forms,

$$
\begin{aligned}
a_{d}^{e}\left(\boldsymbol{\varphi}_{h}, \delta \boldsymbol{\varphi}\right) & =\int_{\mathcal{A}_{e}} \bar{\rho} \ddot{\boldsymbol{\varphi}} \cdot \delta \boldsymbol{\varphi} d \mathcal{A} \\
a_{n}^{e}\left(\boldsymbol{\varphi}_{h}, \delta \boldsymbol{\varphi}\right) & =\int_{\mathcal{A}_{e}} \bar{j}_{0} \boldsymbol{n}^{\alpha}\left(\boldsymbol{\varphi}_{h}\right) \cdot \delta \boldsymbol{\varphi}_{, \alpha} d \mathcal{A}, \\
a_{m}^{e}\left(\boldsymbol{\varphi}_{h}, \delta \boldsymbol{\varphi}\right) & =\int_{\mathcal{A}_{e}} \bar{j}_{0} \tilde{\boldsymbol{m}}^{\alpha}\left(\boldsymbol{\varphi}_{h}\right) \cdot \delta \Delta \boldsymbol{t}_{, \alpha} d \mathcal{A}, \text { and } \\
a_{s}^{e}\left(\boldsymbol{\varphi}_{h}, \delta \boldsymbol{\varphi}\right) & =\int_{\mathcal{A}_{e}}\left(\bar{j}_{0} \boldsymbol{l}\right)_{, \alpha} \cdot \int_{\alpha} \delta \Delta \boldsymbol{t}_{\beta} \boldsymbol{\varphi}_{0}^{, \beta} d \alpha^{\prime} d \mathcal{A}
\end{aligned}
$$

Bi-linear term (27) will be the key term to ensure weakly the continuity of normal displacement as this normal deflection appears explicitly from integration of relation (11) in Eq. (27).

As continuity is not ensured across $\partial_{I} \mathcal{A}_{h}$, jump $\llbracket \bullet \rrbracket$ and mean $\langle\bullet\rangle$ operators are defined on the space of the trace $\mathbf{T R}\left(\partial_{I} \mathcal{A}_{h}\right)=\prod_{e} \mathbf{L}^{2}\left(\partial_{I} \mathcal{A}_{e}\right)$ of vectors that can take multiple values on this boundary, with

$$
\llbracket \bullet \rrbracket=\bullet^{+}-\bullet^{-} \text {, and }\langle\bullet\rangle=\frac{1}{2}\left(\bullet^{+}+\bullet^{-}\right) \text {. }
$$

In these relations the bullets represent generic vector fields with

$$
\bullet^{ \pm}=\lim _{\varepsilon \rightarrow 0^{+}} \bullet\left(\xi^{1} \pm \varepsilon \zeta^{1}, \xi^{2} \pm \varepsilon \zeta^{2}\right)
$$


where $\zeta^{\alpha}$ are the components of the outer unit normal $\boldsymbol{\zeta}$ of $\mathcal{A}_{e}$ in the basis $\boldsymbol{E}_{\alpha}$. These operators lead to vectors that are now belonging to $\mathbf{L}^{2}\left(\partial_{I} \mathcal{A}_{h}\right)$. It is worth noticing that if definition (28) of the jump operator is not independent of the choice of the + and - sides of an element edge, when this jump is used in combination with the outward unit normal of the - element $\boldsymbol{\nu}^{-}$, the formulation becomes consistent and independent on this choice. Although jump and mean operators are mainly meaningful on the interior boundary $\partial_{I} \mathcal{A}$, their definitions can be extended to $\partial_{T} \mathcal{A}$ with

$$
\begin{gathered}
\llbracket \boldsymbol{t} \rrbracket=\overline{\boldsymbol{t}}-\boldsymbol{t}, \llbracket \delta \boldsymbol{t} \rrbracket=-\delta \boldsymbol{t} \text { and }\left\langle\tilde{\boldsymbol{m}}^{\alpha}\right\rangle=\tilde{\boldsymbol{m}}^{\alpha} \text { on } \partial_{T} \mathcal{A}, \\
\llbracket \boldsymbol{\varphi} \rrbracket=\overline{\boldsymbol{\varphi}}-\boldsymbol{\varphi}, \llbracket \delta \boldsymbol{\varphi} \rrbracket=-\delta \boldsymbol{\varphi} \text { and }\left\langle\boldsymbol{n}^{\alpha}\right\rangle=\boldsymbol{n}^{\alpha} \text { on } \partial_{U} \mathcal{A}
\end{gathered}
$$

From these definitions, the boundary terms or Eq. (23) are rewritten

$$
\begin{aligned}
& \sum_{e} \int_{\partial \mathcal{A}_{e}} \bar{j}_{0} \boldsymbol{n}^{\alpha}\left(\boldsymbol{\varphi}_{h}\right) \cdot \delta \boldsymbol{\varphi} \nu_{\alpha} d \mathcal{A}= \\
& \int_{\partial \mathcal{A}_{h}} \bar{j}_{0} \boldsymbol{n}^{\alpha}\left(\boldsymbol{\varphi}_{h}\right) \cdot \delta \boldsymbol{\varphi} \nu_{\alpha} d \mathcal{A}-\int_{\partial_{I} \mathcal{A}_{h}} \llbracket \bar{j}_{0} \boldsymbol{n}^{\alpha}\left(\boldsymbol{\varphi}_{h}\right) \cdot \delta \boldsymbol{\varphi} \rrbracket \nu_{\alpha}^{-} d \mathcal{A}, \\
& \sum_{e} \int_{\partial \mathcal{A}_{e}} \bar{j}_{0} \tilde{\boldsymbol{m}}^{\alpha}\left(\boldsymbol{\varphi}_{h}\right) \cdot \delta \Delta \boldsymbol{t} \nu_{\alpha} d \mathcal{A}= \\
& \int_{\partial \mathcal{A}_{h}} \bar{j}_{0} \tilde{\boldsymbol{m}}^{\alpha}\left(\boldsymbol{\varphi}_{h}\right) \cdot \delta \Delta \boldsymbol{t} \nu_{\alpha} d \mathcal{A}-\int_{\partial_{I} \mathcal{A}_{h}} \llbracket \bar{j}_{0} \tilde{\boldsymbol{m}}^{\alpha}\left(\boldsymbol{\varphi}_{h}\right) \cdot \delta \Delta \boldsymbol{t} \rrbracket \nu_{\alpha}^{-} d \mathcal{A}, \text { and }(33) \\
& -\sum_{e} \int_{\partial \mathcal{A}_{e}} \bar{j}_{0} \boldsymbol{l} \cdot \int_{\alpha} \delta \Delta \boldsymbol{t}_{\beta} \boldsymbol{\varphi}_{0}^{, \beta} d \alpha \nu_{\alpha} d \mathcal{A}= \\
& -\int_{\partial \mathcal{A}_{h}} \bar{j}_{0} \boldsymbol{l} \cdot \int_{\alpha} \delta \Delta \boldsymbol{t}_{\beta} \boldsymbol{\varphi}_{0}^{, \beta} d \alpha \nu_{\alpha} d \mathcal{A}+\int_{\partial_{I} \mathcal{A}_{h}} \llbracket \bar{j}_{0} \boldsymbol{l} \cdot \int_{\alpha} \delta \Delta \boldsymbol{t}_{\beta} \boldsymbol{\varphi}_{0}^{, \beta} d \alpha \rrbracket \nu_{\alpha}^{-} d \mathcal{A}
\end{aligned}
$$

At this stage, the main idea of DG methods, which consists on the substitution of the jumps by consistent numerical fluxes, can be applied

$$
\begin{gathered}
\int_{\partial_{I} \mathcal{A}_{h}} \llbracket \bar{j}_{0} \boldsymbol{n}^{\alpha}\left(\boldsymbol{\varphi}_{h}\right) \cdot \delta \boldsymbol{\varphi} \rrbracket \nu_{\alpha}^{-} d \mathcal{A} \rightarrow \\
\int_{\partial_{I} \mathcal{A}_{h}}\left\{\llbracket \delta \boldsymbol{\varphi} \rrbracket \cdot \boldsymbol{h}\left(\left(\bar{j}_{0} \boldsymbol{n}^{\alpha}\right)^{+},\left(\bar{j}_{0} \boldsymbol{n}^{\alpha}\right)^{-}, \nu_{\alpha}^{-}\right)\right\} d \mathcal{A}, \\
\int_{\partial_{I} \mathcal{A}_{h}} \llbracket \bar{j}_{0} \tilde{\boldsymbol{m}}^{\alpha}\left(\boldsymbol{\varphi}_{h}\right) \cdot \delta \Delta \boldsymbol{t} \rrbracket \nu_{\alpha}^{-} d \mathcal{A} \rightarrow \\
\int_{\partial_{I} \mathcal{A}_{h}}\left\{\llbracket \delta \Delta \boldsymbol{t} \rrbracket \cdot \boldsymbol{h}\left(\bar{j}_{0}\left(\tilde{\boldsymbol{m}}^{\alpha}\right)^{+}, \bar{j}_{0}\left(\tilde{\boldsymbol{m}}^{\alpha}\right)^{-}, \nu_{\alpha}^{-}\right)\right\} d \mathcal{A}, \text { and } \\
\int_{\partial_{I} \mathcal{A}_{h}} \llbracket \bar{j}_{0} \boldsymbol{l} \cdot \int_{\alpha} \delta \Delta \boldsymbol{t}_{\beta} \boldsymbol{\varphi}_{0}^{, \beta} d \alpha \rrbracket \nu_{\alpha}^{-} d \mathcal{A} \rightarrow \\
\int_{\partial_{I} \mathcal{A}_{h}}\left\{\llbracket \int_{\alpha} \delta \Delta \boldsymbol{t}_{\beta} \boldsymbol{\varphi}_{0}^{, \beta} d \alpha \rrbracket \cdot \boldsymbol{h}\left(\left(\bar{j}_{0} \boldsymbol{l}\right)^{+},\left(\bar{j}_{0} \boldsymbol{l}\right)^{-}, \nu_{\alpha}^{-}\right)\right\} d \mathcal{A} .
\end{gathered}
$$


To ensure the consistency of the method, theses fluxes have to verify the following conditions

$$
\begin{aligned}
\boldsymbol{h}\left(\bullet, \bullet, \nu_{\alpha}\right) & =\bullet \nu_{\alpha}^{-} \text {and } \\
\boldsymbol{h}\left((\bullet)^{+},(\bullet)^{-}, \nu_{\alpha}^{-}\right) & =-\boldsymbol{h}\left((\bullet)^{-},(\bullet)^{+}, \nu_{\alpha}^{+}\right),
\end{aligned}
$$

where $\bullet$ represents the quantities $\bar{j}_{0} \boldsymbol{n}^{\alpha}, \bar{j}_{0} \tilde{\boldsymbol{m}}^{\alpha}$ or $\bar{j}_{0} \boldsymbol{l}$. Among the different possibilities, the numerical fluxes chosen in this paper are the traditional average fluxes

$$
\begin{gathered}
\boldsymbol{h}\left(\left(\bar{j}_{0} \boldsymbol{n}^{\alpha}\right)^{+},\left(\bar{j}_{0} \boldsymbol{n}^{\alpha}\right)^{-}, \nu_{\alpha}^{-}\right)=\left\langle\bar{j}_{0} \boldsymbol{n}^{\alpha}\right\rangle \nu_{\alpha}^{-}, \\
\boldsymbol{h}\left(\left(\bar{j}_{0} \tilde{\boldsymbol{m}}^{\alpha}\right)^{+},\left(\bar{j}_{0} \tilde{\boldsymbol{m}}^{\alpha}\right)^{-}, \nu_{\alpha}^{-}\right)=\left\langle\bar{j}_{0} \tilde{\boldsymbol{m}}^{\alpha}\right\rangle \nu_{\alpha}^{-} \text {and } \\
\boldsymbol{h}\left(\left(\bar{j}_{0} \boldsymbol{l}\right)^{+},\left(\bar{j}_{0} \boldsymbol{l}\right)^{-}, \nu_{\alpha}^{-}\right)=\left\langle\bar{j}_{0} \boldsymbol{l}\right\rangle \nu_{\alpha}^{-} .
\end{gathered}
$$

Combining equations (32) - (42), the weak form (23) of the problem becomes

$$
\begin{aligned}
\sum_{e} a_{d}^{e}\left(\boldsymbol{\varphi}_{h}, \delta \boldsymbol{\varphi}\right)= & -\sum_{e} a_{n}^{e}\left(\boldsymbol{\varphi}_{h}, \delta \boldsymbol{\varphi}\right)-\sum_{e} a_{m}^{e}\left(\boldsymbol{\varphi}_{h}, \delta \boldsymbol{\varphi}\right)+\sum_{e} a_{s}^{e}\left(\boldsymbol{\varphi}_{h}, \delta \boldsymbol{\varphi}\right)- \\
& \sum_{s} a_{n I 1}^{s}\left(\boldsymbol{\varphi}_{h}, \delta \boldsymbol{\varphi}\right)-\sum_{s} a_{m I 1}^{s}\left(\boldsymbol{\varphi}_{h}, \delta \boldsymbol{\varphi}\right)+\sum_{s} a_{s I 1}^{s}\left(\boldsymbol{\varphi}_{h}, \delta \boldsymbol{\varphi}\right),
\end{aligned}
$$

with,

$$
\begin{aligned}
a_{n I 1}^{s}\left(\boldsymbol{\varphi}_{h}, \delta \boldsymbol{\varphi}\right) & =\int_{s}\left\langle\bar{j}_{0} \boldsymbol{n}^{\alpha}\right\rangle \cdot \llbracket \delta \boldsymbol{\varphi}_{h} \rrbracket \nu_{\alpha}^{-} d \partial \mathcal{A}_{e}, \\
a_{m I 1}^{s}\left(\boldsymbol{\varphi}_{h}, \delta \boldsymbol{\varphi}\right) & =\int_{s}\left\langle\bar{j}_{0} \tilde{\boldsymbol{m}}^{\alpha}\right\rangle \cdot \llbracket \delta \Delta \boldsymbol{t} \rrbracket \nu_{\alpha}^{-} d \partial \mathcal{A}_{e}, \text { and } \\
a_{s I 1}^{s}\left(\boldsymbol{\varphi}_{h}, \delta \boldsymbol{\varphi}\right) & =\int_{s}\left\langle\bar{j}_{0} \boldsymbol{l}\right\rangle \cdot \llbracket \int_{\alpha} \delta \Delta \boldsymbol{t}_{\beta} \boldsymbol{\varphi}_{0}^{, \beta} d \alpha^{\prime} \rrbracket \nu_{\alpha}^{-} d \partial \mathcal{A}_{e} .
\end{aligned}
$$

To shorten the equations, as it has been previously postulated that there is no external effort, it is also considered here that the boundary integrals on $\partial \mathcal{A}_{h}$ appearing in the set of equations (32 - 34) are equal to zero, and are not reported in (43).

The weak enforcement of the compatibility conditions at interfaces can be achieved with the same approach:

$$
\begin{gathered}
\int_{\partial_{I} \mathcal{A}_{h}} \llbracket \boldsymbol{\varphi} \rrbracket \cdot \boldsymbol{h}\left(\delta\left(\bar{j}_{0} \boldsymbol{n}^{\alpha}\right)^{+}, \delta\left(\bar{j}_{0} \boldsymbol{n}^{\alpha}\right)^{-}, \nu_{\alpha}^{-}\right) d \mathcal{A}=0, \\
\int_{\partial_{I} \mathcal{A}_{h}} \llbracket \boldsymbol{t}\left(\boldsymbol{\varphi}_{h}\right) \rrbracket \cdot \boldsymbol{h}\left(\delta\left(\bar{j}_{0} \tilde{\boldsymbol{m}}^{\alpha}\right)^{+}, \delta\left(\bar{j}_{0} \tilde{\boldsymbol{m}}^{\alpha}\right)^{-}, \nu_{\alpha}^{-}\right) d \mathcal{A}=0, \text { and } \\
\int_{\partial_{I} \mathcal{A}_{h}} \llbracket \int_{\alpha} \Delta \boldsymbol{t}_{\beta} \boldsymbol{\varphi}_{0}^{\beta} d \alpha \rrbracket \cdot \boldsymbol{h}\left(\delta\left(\bar{j}_{0} \boldsymbol{l}\right)^{+}, \delta\left(\bar{j}_{0} \boldsymbol{l}\right)^{-}, \nu_{\alpha}^{-}\right) d \mathcal{A}=0 .
\end{gathered}
$$


In order to develop a symmetric formulation, average fluxes are also selected for these terms, in such a way that equation (43) is reformulated as finding $\varphi_{h} \in \mathrm{U}_{\mathrm{h}}^{k}, \forall \delta \varphi \in \mathrm{U}_{\mathrm{h}_{c}}^{k}$, such that

$$
\begin{aligned}
a_{d}^{e}\left(\boldsymbol{\varphi}_{h}, \delta \boldsymbol{\varphi}\right)= & -\sum_{e} a_{n}^{e}\left(\boldsymbol{\varphi}_{h}, \delta \boldsymbol{\varphi}\right)-\sum_{e} a_{m}^{e}\left(\boldsymbol{\varphi}_{h}, \delta \boldsymbol{\varphi}\right)+\sum_{e} a_{s}^{e}\left(\boldsymbol{\varphi}_{h}, \delta \boldsymbol{\varphi}\right) \\
& -\sum_{s} a_{n I 1}^{s}\left(\boldsymbol{\varphi}_{h}, \delta \boldsymbol{\varphi}\right)-\sum_{s} a_{m I 1}^{s}\left(\boldsymbol{\varphi}_{h}, \delta \boldsymbol{\varphi}\right)+\sum_{s} a_{s I 1}^{s}\left(\boldsymbol{\varphi}_{h}, \delta \boldsymbol{\varphi}\right) \\
& -\sum_{s} a_{n I 2}^{s}\left(\boldsymbol{\varphi}_{h}, \delta \boldsymbol{\varphi}\right)-\sum_{s} a_{m I 2}^{s}\left(\boldsymbol{\varphi}_{h}, \delta \boldsymbol{\varphi}\right)+\sum_{s} a_{s I 2}^{s}\left(\boldsymbol{\varphi}_{h}, \delta \boldsymbol{\varphi}\right),
\end{aligned}
$$

with,

$$
\begin{aligned}
a_{n I 2}^{s}\left(\boldsymbol{\varphi}_{h}, \delta \boldsymbol{\varphi}\right) & =\int_{s} \llbracket \boldsymbol{\varphi}_{h} \rrbracket \cdot\left\langle\boldsymbol{\varphi}_{0, \beta}\left(\mathcal{H}_{n}^{\alpha \beta \gamma \delta} \delta \varepsilon_{\gamma \delta}+\lambda_{\mu}^{\beta} \mathcal{H}_{m}^{\alpha \mu \gamma \delta} \delta \rho_{\gamma \delta}\right) \bar{j}_{0}\right\rangle \nu_{\alpha}^{-} d \partial \mathcal{A}_{e} \\
a_{m I 2}^{s}\left(\boldsymbol{\varphi}_{h}, \delta \boldsymbol{\varphi}\right) & =\int_{s} \llbracket \Delta \boldsymbol{t} \rrbracket \cdot\left\langle\boldsymbol{\varphi}_{0, \beta} \mathcal{H}_{m}^{\alpha \beta \gamma \delta} \delta \rho_{\gamma \delta} \bar{j}_{0}\right\rangle \nu_{\alpha}^{-} d \partial \mathcal{A}_{e}, \text { and } \\
a_{s I 2}^{s}\left(\boldsymbol{\varphi}_{h}, \delta \boldsymbol{\varphi}\right) & =\int_{s} \llbracket \int_{\alpha} \Delta \boldsymbol{t}_{\beta} \boldsymbol{\varphi}_{0}^{\beta} d \alpha^{\prime} \rrbracket \cdot\left\langle\boldsymbol{\varphi}_{0, \gamma} \mathcal{H}_{s}^{\gamma \delta} \delta \gamma_{\delta} \bar{j}_{0}\right\rangle \nu_{\alpha}^{-} d \partial \mathcal{A}_{e}
\end{aligned}
$$

where Eq. (51) is deduced from (7), with the Kirchhoff-Love assumption.

Finally as it is well known that, for elliptic problems, such a formulation is unstable, the method is stabilized by introducing quadratic terms as it is suggested in $[30,34,35]$. Such an introduction of interior penalty term is usual for the DG method applied to solid mechanics (see [40-45] among others). Although the DG method is now slightly dissipative, this does not impact on the numerical accuracy as the method remains consistent and converges toward the solution with an optimal rate. These terms depend on dimensionless stabilization parameters $\beta_{i}$, which are sufficiently large constants, and which are independent of the mesh and material properties. The mesh independence is ensured by the introduction of the characteristic element size $h^{s}$ in the stability terms. The introduction of stabilization parameters in (50) gives the final expression of the problem, which is finding $\varphi_{h} \in \mathrm{U}_{\mathrm{h}}^{k}, \forall \delta \varphi \in \mathrm{U}_{\mathrm{h}_{c}}^{k}$, such that,

$$
\begin{aligned}
a_{d}^{e}\left(\boldsymbol{\varphi}_{h}, \delta \boldsymbol{\varphi}\right)= & -\sum_{e} a_{n}^{e}\left(\boldsymbol{\varphi}_{h}, \delta \boldsymbol{\varphi}\right)-\sum_{e} a_{m}^{e}\left(\boldsymbol{\varphi}_{h}, \delta \boldsymbol{\varphi}\right)+\sum_{e} a_{s}^{e}\left(\boldsymbol{\varphi}_{h}, \delta \boldsymbol{\varphi}\right) \\
& -\sum_{s} a_{n I 1}^{s}\left(\boldsymbol{\varphi}_{h}, \delta \boldsymbol{\varphi}\right)-\sum_{s} a_{m I 1}^{s}\left(\boldsymbol{\varphi}_{h}, \delta \boldsymbol{\varphi}\right)+\sum_{s} a_{s I 1}^{s}\left(\boldsymbol{\varphi}_{h}, \delta \boldsymbol{\varphi}\right) \\
& -\sum_{s} a_{n I 2}^{s}\left(\boldsymbol{\varphi}_{h}, \delta \boldsymbol{\varphi}\right)-\sum_{s} a_{m I 2}^{s}\left(\boldsymbol{\varphi}_{h}, \delta \boldsymbol{\varphi}\right)+\sum_{s} a_{s I 2}^{s}\left(\boldsymbol{\varphi}_{h}, \delta \boldsymbol{\varphi}\right) \\
& -\sum_{s} a_{n I 3}^{s}\left(\boldsymbol{\varphi}_{h}, \delta \boldsymbol{\varphi}\right)-\sum_{s} a_{m I 3}^{s}\left(\boldsymbol{\varphi}_{h}, \delta \boldsymbol{\varphi}\right)+\sum_{s} a_{s I 3}^{s}\left(\boldsymbol{\varphi}_{h}, \delta \boldsymbol{\varphi}\right),
\end{aligned}
$$


with,

$$
\begin{aligned}
a_{n I 3}^{s}\left(\boldsymbol{\varphi}_{h}, \delta \boldsymbol{\varphi}\right)= & \int_{s} \llbracket \boldsymbol{\varphi}_{h} \rrbracket \cdot \boldsymbol{\varphi}_{0, \gamma} \nu_{\delta}^{-}\left\langle\frac{\beta_{2} \mathcal{H}_{n}^{\alpha \beta \gamma \delta} \bar{j}_{0}}{h^{s}}\right\rangle \llbracket \delta \boldsymbol{\varphi} \rrbracket \cdot \boldsymbol{\varphi}_{0, \beta} \nu_{\alpha}^{-} d \partial \mathcal{A}_{e}+ \\
& \int_{s} \llbracket \boldsymbol{\varphi}_{h} \rrbracket \cdot \boldsymbol{\varphi}_{0, \gamma} \nu_{\delta}^{-}\left\langle\lambda_{\mu}^{\beta} \frac{\beta_{2} \mathcal{H}_{m}^{\alpha \mu \gamma \delta} \bar{j}_{0}}{h^{s}}\right\rangle \llbracket \delta \Delta \boldsymbol{t} \rrbracket \cdot \boldsymbol{\varphi}_{0, \beta} \nu_{\alpha}^{-} d \partial \mathcal{A}_{e}+ \\
& \int_{s} \llbracket \Delta \boldsymbol{t} \rrbracket \cdot \boldsymbol{\varphi}_{0, \gamma} \nu_{\delta}^{-}\left\langle\lambda_{\mu}^{\beta} \frac{\beta_{2} \mathcal{H}_{m}^{\alpha \mu \gamma \delta} \bar{j}_{0}}{h^{s}}\right\rangle \llbracket \delta \boldsymbol{\varphi}_{h} \rrbracket \cdot \boldsymbol{\varphi}_{0, \beta} \nu_{\alpha}^{-} d \partial \mathcal{A}_{e} \\
a_{m I 3}^{s}\left(\boldsymbol{\varphi}_{h}, \delta \boldsymbol{\varphi}\right)= & \int_{s} \llbracket \Delta \boldsymbol{t} \rrbracket \cdot \boldsymbol{\varphi}_{0, \gamma} \nu_{\delta}^{-}\left\langle\frac{\beta_{1} \mathcal{H}_{m}^{\alpha \beta \gamma \delta} \bar{j}_{0}}{h^{s}}\right\rangle \llbracket \delta \Delta \boldsymbol{t} \rrbracket \cdot \boldsymbol{\varphi}_{0, \beta} \nu_{\alpha}^{-} d \partial \mathcal{A}_{e}, \\
a_{s I 3}^{s}\left(\boldsymbol{\varphi}_{h}, \delta \boldsymbol{\varphi}\right)= & \int_{s}\left\{\llbracket \int_{\mu} \Delta \boldsymbol{t}_{\gamma} \boldsymbol{\varphi}_{0}^{, \gamma} d \mu^{\prime} \rrbracket \cdot \boldsymbol{\varphi}_{0, \alpha} \nu_{\mu}^{-}\left\langle\frac{\beta_{3} \mathcal{H}_{s}^{\alpha \beta} \bar{j}_{0}}{h^{s}}\right\rangle\right. \\
& \left.\boldsymbol{\varphi}_{0, \beta} \cdot \llbracket \int_{\nu} \delta \Delta \boldsymbol{t}_{\delta} \boldsymbol{\varphi}_{0}^{, \delta} d \nu^{\prime} \rrbracket \nu_{\nu}^{-}\right\} d \partial \mathcal{A}_{e} .
\end{aligned}
$$

In relation (55), the two first terms result from definition (7), and the last term is introduced to obtain a symmetric stabilization term. We can now take advantage of the Kirchhoff-Love assumption, for which shearing can be neglected. Therefore consistency terms $a_{s I 1}^{s}\left(\varphi_{h}, \delta \varphi\right)(46)$ and compatibility terms $a_{s I 2}^{s}\left(\boldsymbol{\varphi}_{h}, \delta \boldsymbol{\varphi}\right)(53)$ involving $\boldsymbol{l}$ both vanish.

The only terms related to shearing which are remaining are the stability terms (57). These terms will weakly ensure the compatibility of the deflection normal to the mid-surface. However, in order to implement them in an efficient way, it is necessary to find an expression for the primitive of $\Delta \boldsymbol{t}$ and $\delta \Delta t$. Using relation (11) yields

$$
\int_{\mu} \Delta \boldsymbol{t}_{\gamma} \boldsymbol{\varphi}_{0}^{, \gamma} d \mu^{\prime}=-\int_{\mu} \boldsymbol{u}_{h, \gamma} \cdot \boldsymbol{t}_{0} \boldsymbol{\varphi}_{0}^{, \gamma} d \mu^{\prime}
$$

As these terms have as unique purpose the weak enforcement of the stability, they can be approximated without damaging the accuracy of the method. Therefore, these integrals can be approximated by assuming a surface with a curvature radius large compared to the sizes, thus leading for a planar assumption to

$$
\llbracket \int_{\mu} \Delta \boldsymbol{t}_{\gamma} \boldsymbol{\varphi}_{0}^{, \gamma} d \mu^{\prime} \rrbracket \cdot \boldsymbol{\varphi}_{0, \alpha}=-\llbracket \int_{\mu} \boldsymbol{\varphi}_{h, \gamma} \cdot \boldsymbol{t}_{0} \boldsymbol{\varphi}_{0}^{, \gamma} d \mu^{\prime} \rrbracket \cdot \boldsymbol{\varphi}_{0, \alpha} \rightarrow-\llbracket \boldsymbol{\varphi}_{h} \rrbracket \cdot \boldsymbol{t}_{0} \delta_{\alpha \mu} .
$$

Therefore, the expression of $a_{n I 3}^{s}\left(\boldsymbol{\varphi}_{h}, \delta \boldsymbol{\varphi}\right)(55)$ can be simplified accordingly, by neglecting the curvature term, leading to

$$
a_{n I 3}^{s}\left(\boldsymbol{\varphi}_{h}, \delta \boldsymbol{\varphi}\right)=\int_{s} \llbracket \varphi_{h} \rrbracket \cdot \varphi_{0, \gamma} \nu_{\delta}^{-}\left\langle\frac{\beta_{2} \mathcal{H}_{n}^{\alpha \beta \gamma \delta} \bar{j}_{0}}{h^{s}}\right\rangle \llbracket \delta \varphi \rrbracket \cdot \varphi_{0, \beta} \nu_{\alpha}^{-} d \partial \mathcal{A}_{e} \cdot
$$


Owing to these last considerations, weak form (54) is stated as finding $\boldsymbol{\varphi}_{h} \in$ $\mathrm{U}_{\mathrm{h}}^{k}, \forall \delta \varphi \in \mathrm{U}_{\mathrm{h} c}^{k}$, such that,

$$
\begin{aligned}
a\left(\boldsymbol{\varphi}_{h}, \delta \boldsymbol{\varphi}\right)= & \sum_{e}\left(a_{d}^{e}\left(\boldsymbol{\varphi}_{h}, \delta \boldsymbol{\varphi}\right)+a_{n}^{e}\left(\boldsymbol{\varphi}_{h}, \delta \boldsymbol{\varphi}\right)+a_{m}^{e}\left(\boldsymbol{\varphi}_{h}, \delta \boldsymbol{\varphi}\right)\right)+ \\
& \sum_{s}\left(a_{n I}^{s}\left(\boldsymbol{\varphi}_{h}, \delta \boldsymbol{\varphi}\right)+a_{m I}^{s}\left(\boldsymbol{\varphi}_{h}, \delta \boldsymbol{\varphi}\right)-a_{s I}^{s}\left(\boldsymbol{\varphi}_{h}, \delta \boldsymbol{\varphi}\right)\right)=0
\end{aligned}
$$

with

$$
\begin{aligned}
a_{n I}^{s}\left(\boldsymbol{\varphi}_{h}, \delta \boldsymbol{\varphi}\right) & =a_{n I 1}^{s}\left(\boldsymbol{\varphi}_{h}, \delta \boldsymbol{\varphi}\right)+a_{n I 2}^{s}\left(\boldsymbol{\varphi}_{h}, \delta \boldsymbol{\varphi}\right)+a_{n I 3}^{s}\left(\boldsymbol{\varphi}_{h}, \delta \boldsymbol{\varphi}\right), \\
a_{m I}^{s}\left(\boldsymbol{\varphi}_{h}, \delta \boldsymbol{\varphi}\right) & =a_{m I 1}^{s}\left(\boldsymbol{\varphi}_{h}, \delta \boldsymbol{\varphi}\right)+a_{m I 2}^{s}\left(\boldsymbol{\varphi}_{h}, \delta \boldsymbol{\varphi}\right)+a_{m I 3}^{s}\left(\boldsymbol{\varphi}_{h}, \delta \boldsymbol{\varphi}\right), \text { and }(63) \\
a_{s I}^{s}\left(\boldsymbol{\varphi}_{h}, \delta \boldsymbol{\varphi}\right) & =\int_{s} \llbracket \boldsymbol{\varphi}_{h} \rrbracket \cdot \boldsymbol{t}_{0} \nu_{\beta}^{-}\left\langle\frac{\beta_{3} \mathcal{H}_{s}^{\alpha \beta} \bar{j}_{0}}{h^{s}}\right\rangle \llbracket \delta \boldsymbol{\varphi} \rrbracket \cdot \boldsymbol{t}_{0} \nu_{\alpha}^{-} d \partial \mathcal{A}_{e} .
\end{aligned}
$$

Furthermore, if continuous test and trial functions are used, $\llbracket \boldsymbol{\varphi}_{h} \rrbracket=\llbracket \delta \varphi \rrbracket=$ 0 and for a quasi-static problem (ie $a_{d}^{e}\left(\boldsymbol{\varphi}_{h}, \delta \boldsymbol{\varphi}\right)=0$ ), equation (61) can be simplified into

$$
a\left(\boldsymbol{\varphi}_{h}, \delta \boldsymbol{\varphi}\right)=\sum_{e}\left(a_{n}^{e}\left(\boldsymbol{\varphi}_{h}, \delta \boldsymbol{\varphi}\right)+a_{m}^{e}\left(\boldsymbol{\varphi}_{h}, \delta \boldsymbol{\varphi}\right)\right)+\sum_{s} a_{m I}^{s}\left(\boldsymbol{\varphi}_{h}, \delta \boldsymbol{\varphi}\right)=0
$$

which is identical to the $C^{0} / \mathrm{DG}$ formulation presented in [34].

The final resulting bi-linear form $a\left(\boldsymbol{\varphi}_{h}, \delta \boldsymbol{\varphi}\right)(61)$ of the problem contains the classical terms of shell theory $a_{n}^{e}\left(\boldsymbol{\varphi}_{h}, \delta \boldsymbol{\varphi}\right)$ and $a_{m}^{e}\left(\boldsymbol{\varphi}_{h}, \delta \boldsymbol{\varphi}\right)$, while the third term is a collection of boundary integrals resulting from the inter-element discontinuities. They enforce respectively

- the consistency of the formulation for $a_{n I 1}^{s}\left(\boldsymbol{\varphi}_{h}, \delta \boldsymbol{\varphi}\right)$ and $a_{m I 1}^{s}\left(\boldsymbol{\varphi}_{h}, \delta \boldsymbol{\varphi}\right)$,

- the symmetric nature of the Jacobian for $a_{n I 2}^{s}\left(\varphi_{h}, \delta \boldsymbol{\varphi}\right)$ and $a_{m I 2}^{s}\left(\varphi_{h}, \delta \boldsymbol{\varphi}\right)$, and

- the stability for $a_{n I 3}^{s}\left(\boldsymbol{\varphi}_{h}, \delta \boldsymbol{\varphi}\right), a_{m I 3}^{s}\left(\boldsymbol{\varphi}_{h}, \delta \boldsymbol{\varphi}\right)$ and $a_{s I}^{s}\left(\boldsymbol{\varphi}_{h}, \delta \boldsymbol{\varphi}\right)$.

\subsection{Remark on the bi-linear form}

Bulk terms $a_{n}^{e}\left(\boldsymbol{\varphi}_{h}, \delta \boldsymbol{\varphi}\right)$ and $a_{m}^{e}\left(\boldsymbol{\varphi}_{h}, \delta \boldsymbol{\varphi}\right)$ of bi-linear form (61) can be rewritten in the context of small deformations in a more familiar form as obtained by [38]. Indeed, from Eqs. (25-26),

$$
\begin{aligned}
& a_{n}^{e}\left(\boldsymbol{\varphi}_{h}, \delta \boldsymbol{\varphi}\right)+a_{m}^{e}\left(\boldsymbol{\varphi}_{h}, \delta \boldsymbol{\varphi}\right)= \\
& \quad \int_{\mathcal{A}_{e}}\left(\bar{j}_{0} \tilde{n}^{\alpha \beta} \boldsymbol{\varphi}_{0, \beta} \cdot \delta \boldsymbol{\varphi}, \alpha+\bar{j}_{0} \lambda_{\mu}^{\beta} \tilde{m}^{\alpha \mu} \boldsymbol{\varphi}_{0, \beta} \cdot \delta \boldsymbol{\varphi}_{, \alpha}+\bar{j}_{0} \tilde{m}^{\alpha \beta} \boldsymbol{\varphi}_{0, \beta} \cdot \delta \Delta \boldsymbol{t}_{, \alpha}\right) d \mathcal{A} .
\end{aligned}
$$


where definitions (7-9) have been particularized to the Kirchhoff-Love assumption. As $\lambda_{\mu}^{\beta} \boldsymbol{\varphi}_{0, \beta}=\boldsymbol{t}_{0, \mu}$ by definition, Eq. (66) can be rewritten

$$
\begin{aligned}
& a_{n}^{e}\left(\boldsymbol{\varphi}_{h}, \delta \boldsymbol{\varphi}\right)+a_{m}^{e}\left(\boldsymbol{\varphi}_{h}, \delta \boldsymbol{\varphi}\right)= \\
& \int_{\mathcal{A}_{e}}\left(\bar{j}_{0} \tilde{n}^{\alpha \beta} \boldsymbol{\varphi}_{0, \beta} \cdot \delta \boldsymbol{\varphi}_{, \alpha}+\bar{j}_{0} \tilde{m}^{\alpha \beta}\left(\boldsymbol{t}_{0, \beta} \cdot \delta \boldsymbol{\varphi}_{, \alpha}+\boldsymbol{\varphi}_{0, \beta} \cdot \delta \Delta \boldsymbol{t}, \alpha\right)\right) d \mathcal{A}= \\
& \quad \int_{\mathcal{A}_{e}}\left(\bar{j}_{0} \tilde{n}^{\alpha \beta} \delta \varepsilon_{\alpha \beta}+\bar{j}_{0} \tilde{m}^{\alpha \beta} \delta \rho_{\alpha \beta}\right) d \mathcal{A},
\end{aligned}
$$

where the definition of the resulting strains (10-12) have been used.

\subsection{Numerical properties}

The presented framework satisfies two fundamental properties of a numerical method: consistency and stability. The last one can be demonstrated if the parameters $\beta_{1}$ and $\beta_{2}$ are large enough, if $\beta_{3}$ is non-zero, and with the use of the approximation (59). As it is lengthly discussed in [30] for beams, in order to reduce locking and to preserve stability, the optimal value for $\beta_{3}$ is $\beta_{1}\left(\frac{h}{L_{c}}\right)^{2}$, where $h$ is the thickness of shell and $L_{c}$ is a characteristic length depending on the problem. The convergence rate of the method in the energy norm with respect to the mesh size is proved to be equal to $k-1$, with $k$ as the degree of the polynomial approximation. Finally, the method presents an optimal-convergence rate $k+1$ in the $\mathrm{L}^{2}$-norm, which can be demonstrated for at least cubic elements. Demonstrations of these properties are summarized in Appendix A

Locking inherent to shell formulations can be avoided owing to the weak enforcement of continuity resulting from the DG method ${ }^{5}$. In particular there is no locking for quadratic elements with reduced integration, and for cubic elements with full integration [34].

\subsection{Numerical example}

The numerical properties of the method are now illustrated on a numerical example. This example considers a pinched open hemisphere, with radius $R$, thickness $t$ and an opening of a spherical sector angle $\theta$ (see values given in Table 1). It is subjected to radial loads $P$ applied on two diametrical directions, see Fig. 3(a). The load is compressive in the $\mathrm{y}$-direction and tensile in the $\mathrm{x}$ direction. One quarter of the structure is modeled by exploiting the symmetries of the problem. The maximal deflection along $\mathrm{x}$ or $\mathrm{y}$ can be computed by the formula: $\left|\delta_{x}\right|=\left|\delta_{y}\right|=0.093\left(\mathrm{mN}^{-1}\right) \frac{P}{2}$ (see [37]) and is used as the reference value. This problem is simulated with 9-node bi-quadratic elements with reduced integration (4 Gauss Points on each shell element and 2 on each interface element) (Q9RI) for several meshes $2 \times 2,4 \times 4,8 \times 8, \ldots, 32 \times 32$ elements and for different values of stability parameters $\beta_{1}=\beta_{2}=\beta_{3}\left(\frac{R}{h}\right)^{2}=10,1 e^{3}, 1 e^{6}$. 


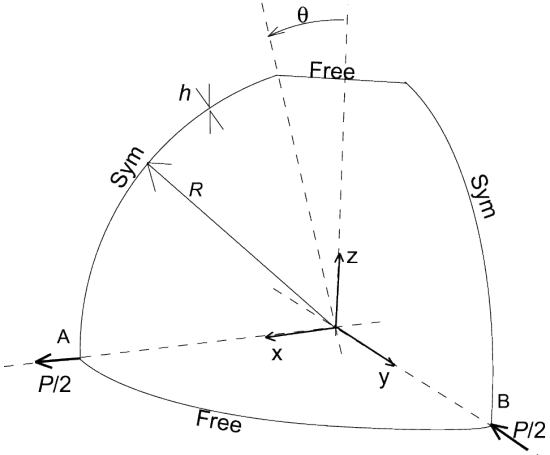

(a)

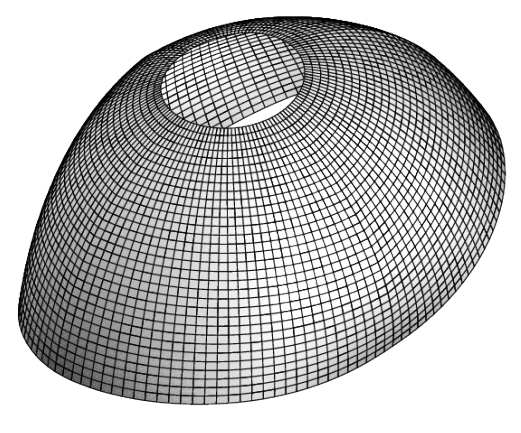

(b)

Figure 3: Study of the pinched open hemisphere. Problem dimensions: radius $R$, thickness $t$, opening $\theta$, concentrated loading $P$. (a) Geometry of the open hemisphere. One fourth is considered. (b) Magnified deformation of the completed hemisphere for a regular mesh of thirty-two quadratic quadrangular elements on each side.

Table 1: Material and geometrical properties for the pinched open hemisphere test.

\begin{tabular}{ll}
\hline Property & Value \\
\hline Radius $R[\mathrm{~m}]$ & 10 \\
Thickness $h[\mathrm{~m}]$ & 0.04 \\
Opening $\theta\left[^{\circ}\right]$ & 18 \\
Young modulus [MPa] & 68.25 \\
Poisson's ratio [-] & 0.3 \\
Applied force $P[\mathrm{~N}]$ & 20 \\
\hline
\end{tabular}

Results obtain by the presented full-DG formulation are compared to the formulation $C^{0} / \mathrm{DG}$ previously presented in [34]. As it can be seen on Fig. 4 , there is no noticeable difference for reduced mesh size. The displacements (Fig. 4(a \& c)), and convergence order (Fig. 4(b \& d)) are the same for both methods. ${ }^{6}$. Note that for the lowest value of stability parameters, the convergence is not monotonic as it can be seen on Fig. $4 \mathrm{c}$ ) and d). This is due to the fact that $\beta_{1}=10$ is close to the stability limit, and thus stability is only ensured for fine-enough meshes.

The $C^{0} /$ DG method for Kirchhoff-Love shells is a displacements-only based

\footnotetext{
${ }^{5}$ Large values of stability parameters can leads to lost of its property.

${ }^{6}$ Note that even if the formula (A.15) predicts a convergence in $k$ for $k=2$ it is numerically observed that the convergence is in $k+1$ (see [30, 31, 34])
} 


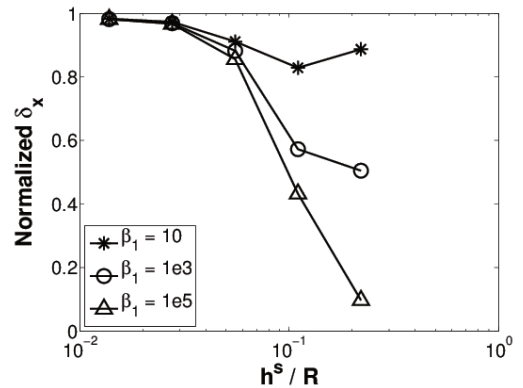

(a)

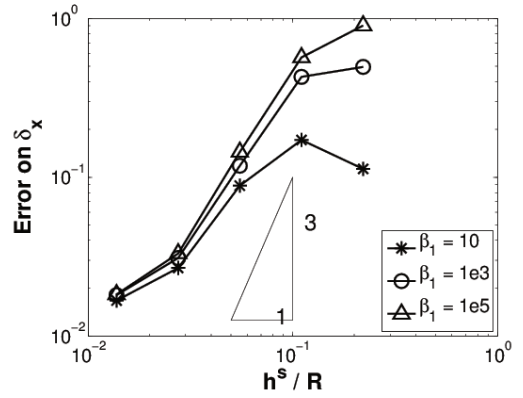

(c)

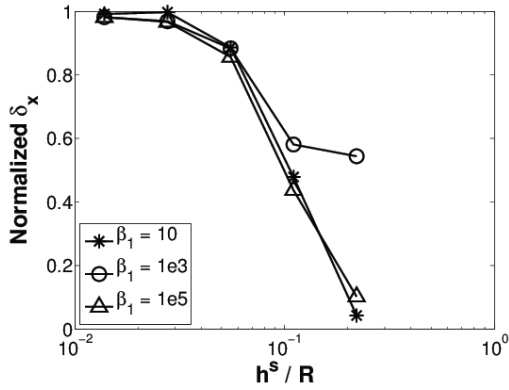

(b)

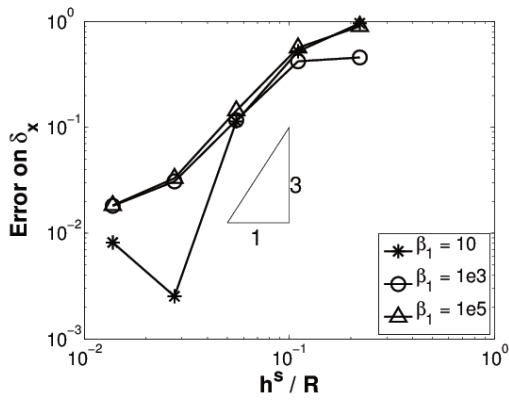

(d)

Figure 4: Influence of mesh size and stabilization parameters on the deflection for the pinched open hemisphere. (a) Normalized deflection for Q9RI with $C^{0} / \mathrm{DG}$ formulation. (b) Normalized deflection for Q9RI with full-DG formulation. (c) Error on the deflection for Q9RI with $C^{0} / \mathrm{DG}$ formulation. (d) Error on the deflection for Q9RI with full-DG formulation.

method, and is particularly attractive as it allows reducing the number of degrees-of-freedom per node compared to usual mixed shell formulations, which consider rotational dofs. However, the full DG formulation requires new degrees of freedom as they have to be distinct for each element attached to one node (or to duplicate the node per elements). For example, with the finest quadratic mesh of this application, the number of unknowns increases from 12675 for the $C^{0} / \mathrm{DG}$ formulation to 27648 for the full-DG formulation. Nevertheless, both methods are one-field formulation, which allows reducing the number of dofs per node compared to classical shell elements. Of course, the full-DG formulation presents an interest only for problems involving the presence of fracture or to guarantee continuity at the interface of mesh partitions in case of parallel simulations [12].

This example demonstrates that it is possible to model a shell continuum mechanical problem with full discontinuous elements in a consistent and stable manner. 


\section{Application to fracture mechanics}

This section explains how the previous full-DG framework can be easily and efficiently combined with an extrinsic cohesive model to solve fracture problems.

\subsection{Combination full-DG / extrinsic cohesive law}

The idea of combining a full-DG method with an extrinsic cohesive law was pioneered by J. Mergheim et al [22] and by R. Radovitzky et al [11, 12] in order to avoid the difficulties inherent to the classical cohesive approaches. The main idea of this method can be summarized by the following equation:

$$
\sum_{e} a_{\mathrm{bulk}}^{e}\left(\boldsymbol{\varphi}_{h}, \delta \boldsymbol{\varphi}\right)+\sum_{s}\left[\left(1-\alpha_{s}\right) a_{\text {inter }}^{s}\left(\boldsymbol{\varphi}_{h}, \delta \boldsymbol{\varphi}\right)+\alpha_{s} a_{\text {cohesive }}^{s}\left(\llbracket \boldsymbol{\varphi}_{h} \rrbracket, \llbracket \delta \boldsymbol{\varphi} \rrbracket\right)\right]
$$

with,

$$
\begin{aligned}
a_{\text {bulk }}^{e}\left(\boldsymbol{\varphi}_{h}, \delta \boldsymbol{\varphi}\right)= & a_{d}^{e}\left(\boldsymbol{\varphi}_{h}, \delta \boldsymbol{\varphi}\right)+a_{n}^{e}\left(\boldsymbol{\varphi}_{h}, \delta \boldsymbol{\varphi}\right)+a_{m}^{e}\left(\boldsymbol{\varphi}_{h}, \delta \boldsymbol{\varphi}\right) \\
a_{\text {inter }}^{s}\left(\boldsymbol{\varphi}_{h}, \delta \boldsymbol{\varphi}\right)= & a_{n I 1}^{s}\left(\boldsymbol{\varphi}_{h}, \delta \boldsymbol{\varphi}\right)+a_{n I 2}^{s}\left(\boldsymbol{\varphi}_{h}, \delta \boldsymbol{\varphi}\right)+a_{n I 3}^{s}\left(\boldsymbol{\varphi}_{h}, \delta \boldsymbol{\varphi}\right)+ \\
& a_{m I 1}^{s}\left(\boldsymbol{\varphi}_{h}, \delta \boldsymbol{\varphi}\right)+a_{m I 2}^{s}\left(\boldsymbol{\varphi}_{h}, \delta \boldsymbol{\varphi}\right)+a_{m I 3}^{s}\left(\boldsymbol{\varphi}_{h}, \delta \boldsymbol{\varphi}\right)- \\
& a_{s I 3}^{s}\left(\boldsymbol{\varphi}_{h}, \delta \boldsymbol{\varphi}\right), \text { and }
\end{aligned}
$$

$a_{\text {cohesive }}^{s}\left(\llbracket \varphi_{h} \rrbracket, \llbracket \delta \varphi \rrbracket\right)$ the bi-linear form of the cohesive terms that has to be defined.

In Eq. (68), $\alpha_{s}$ is a Boolean value, which switches from "false" to "true"

when a fracture criterion is met. Indeed, before onset of fracture, Eq. (68) corresponds to the weak form of the shell problem (61), and thus inherits from his numerical properties of consistency and stability. Upon onset of fracture, the interface terms related to the DG framework are replaced by an extrinsic cohesive law, which has still to be defined. Note that in practice the Boolean $\alpha_{s}$ is evaluated at each Gauss points of interface elements and therefore all Gauss' points of an element are not necessarily fractured.

As it is discussed by the authors in [30] for beams, when considering the theory of thin structures, it is very difficult to separate the thickness part under tension from the part under compression, during a through-the-thickness crack propagation. So it was suggested with success in [30] to apply the cohesive principle to the resultant stresses $\boldsymbol{n}^{\alpha}$ and $\tilde{\boldsymbol{m}}^{\alpha}$, which appear in thin bodies. The same concept is extended here to take into account a mode I or II fracture, or a combination of modes I and II simultaneously.

Toward this end, an effective opening is defined in this paper for each mode and the combination of two modes is performed by following the idea suggested by Camacho et al in [46]. Note that in Kirchhoff-Love theory the out-of-plane shearing is neglected, which implies the impossibility to take into account a fracture in mode III.

Before developing the cohesive law, we will assume that the convected basis, tangential to the shell, at the interface element obeys the following rule: vector $\boldsymbol{\varphi}_{0,1}$ is parallel to the interface element and $\boldsymbol{\varphi}_{0,2}$ is perpendicular to the interface element, as it is illustrated on Fig. 5. 


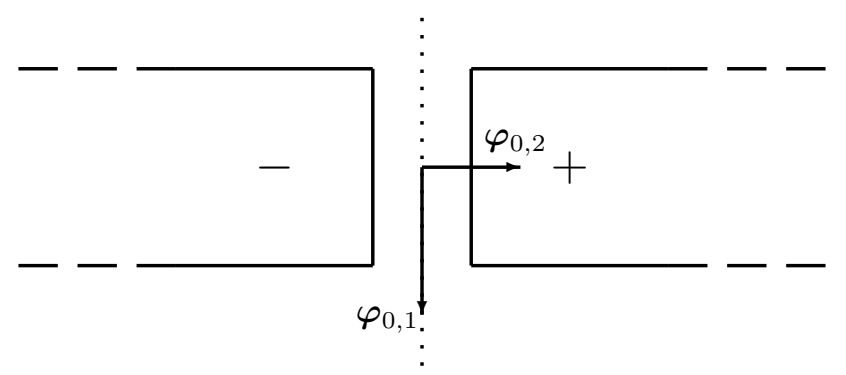

Figure 5: Local basis vector on interface element tangential o the shell. The interface is drawn with dotted line. By convention, $\boldsymbol{\varphi}_{0,1}$ is parallel and $\boldsymbol{\varphi}_{0,2}$ is normal to the interface.

\subsection{Mode I}

Let us first discuss the case of the mode I opening, see Fig. 6. Considering the basis of the interface element as shown on Fig. 5, the resulting efforts are related to an effective opening whose aim is to consider the two parts (tension and bending) of the normal opening represented on Fig. 6.

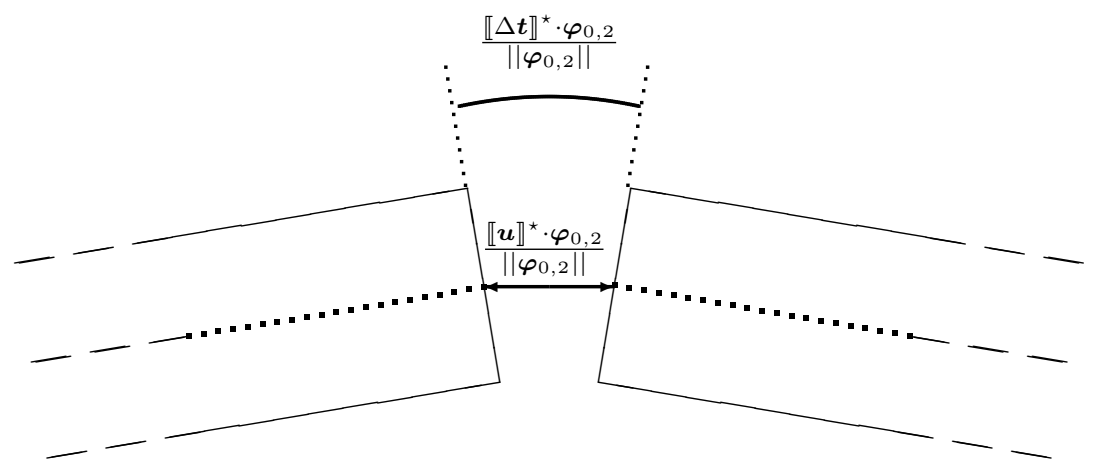

Figure 6: The two components of the normal opening $\Delta_{n}^{\star}$, view perpendicular to the shell. The neutral axis is drawn with a dotted line.

Similarly to what has been suggested in [30], an original normal effective opening, corresponding to the mode I opening, is deduced from the tension and bending openings

$$
\Delta_{n}^{\star}=\left(1-\eta_{I}\right) \frac{\llbracket \boldsymbol{u} \rrbracket^{\star} \cdot \boldsymbol{\varphi}_{0,2}}{\left\|\varphi_{0,2}\right\|} \pm \eta_{I} \frac{h}{6} \frac{\llbracket \Delta \boldsymbol{t} \rrbracket^{\star} \cdot \boldsymbol{\varphi}_{0,2}}{\left\|\boldsymbol{\varphi}_{0,2}\right\|} .
$$

In this expression $\llbracket \boldsymbol{u} \rrbracket^{\star}$ and $\llbracket \Delta \boldsymbol{t} \rrbracket^{\star}$ are respectively the effective openings in displacement and in rotation, resulting from the use of a DG method before fracture activation. Indeed at fracture initialization the opening in displacement $\llbracket \boldsymbol{u} \rrbracket$ and $\llbracket \Delta \boldsymbol{t} \rrbracket$ are not exactly equal to zero due to the weak enforcement of 


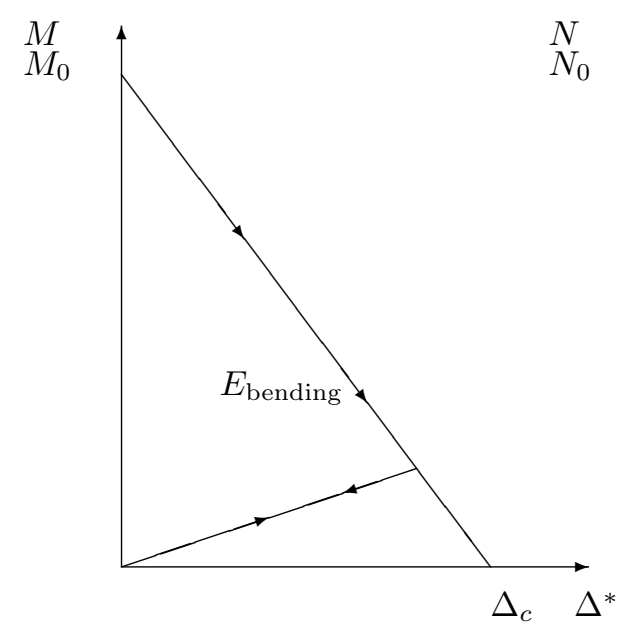

(a)

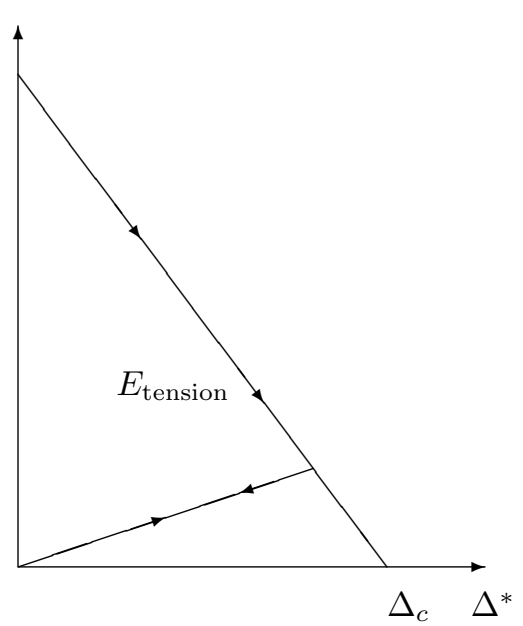

(b)

Figure 7: Linearly decreasing monotonic law for (a) resultant bending stress and for (b) resultant membrane stress.

compatibility. In order to have null openings at fracture initialization these initial values $\llbracket \boldsymbol{u} \rrbracket_{0}$ and $\llbracket \Delta \boldsymbol{t} \rrbracket_{0}$ are subtracted from $\llbracket \boldsymbol{u} \rrbracket$ and $\llbracket \Delta \boldsymbol{t} \rrbracket$, yielding

$$
\begin{aligned}
\llbracket \boldsymbol{u} \rrbracket^{\star} & =\llbracket \boldsymbol{u} \rrbracket-\llbracket \boldsymbol{u} \rrbracket_{0}, \\
\llbracket \Delta \boldsymbol{t} \rrbracket^{\star} & =\llbracket \Delta \boldsymbol{t} \rrbracket-\llbracket \Delta \boldsymbol{t} \rrbracket_{0} .
\end{aligned}
$$

Moreover, the parameter $\eta_{I}$ ensures that the coupling between the resulting tensile stress and the resulting bending stress respects the energetic balance ( $i e$ that the fracture process releases the correct energy), which is obtained for

$$
\eta_{I}=\frac{\frac{6}{h}\left|\left\langle\tilde{m}_{0}^{22}\right\rangle\right|}{\left\langle n_{0}^{22}\right\rangle+\frac{6}{h}\left|\left\langle\tilde{m}_{0}^{22}\right\rangle\right|}
$$

where $n_{0}^{22}$ and $\tilde{m}_{0}^{22}$ are respectively the traction effort and the bending couple at fracture initialization. Indeed, the introduction of the factor $\frac{h}{6}$ in equation (71) ensures the respect of energetic balance, as it is shown here below for a pure bending case. Considering the linearly decreasing cohesive law as on Fig. $7^{7}$

$$
\left\langle\tilde{m}^{22}\right\rangle_{\mathrm{coh}}=\tilde{m}_{0}^{22}\left(1-\frac{\Delta^{\star}}{\Delta_{c}}\right) \frac{\Delta_{n}^{\star}}{\Delta^{\star}},
$$

knowing the critical bending stress in pure bending

$$
\tilde{m}_{0}^{22}=\frac{h^{2} \sigma_{c}}{6}
$$

\footnotetext{
${ }^{7}$ The demonstration remains valid with another cohesive law.
} 
and using the definition of the resulting normal opening (71) lead to

$$
\begin{aligned}
\int_{0}^{\Delta_{r c}} M\left(\Delta_{n}^{\star}\right) d \llbracket \Delta \boldsymbol{t} \rrbracket & =\int_{0}^{\Delta_{c}} \pm \frac{6}{h}\left\langle\tilde{m}_{0}^{22}\right\rangle\left(1-\frac{\Delta_{n}^{\star}}{\Delta_{c}}\right) d \Delta_{n}^{\star} \\
& =\frac{6}{h} \frac{h^{2} \sigma_{c}}{6} \frac{\Delta_{c}}{2}=h G_{c},
\end{aligned}
$$

where $\Delta_{r c}=\frac{h}{6} \frac{\llbracket \Delta t \rrbracket_{c}^{\star} \cdot \varphi_{0,2}}{\left\|\varphi_{0.2}\right\|}$ is the critical opening in rotation, for which the fracture process is completed, where $G_{c}$ and $\sigma_{\mathrm{c}}$ are respectively the fracture energy and a spall stress depending on the material only, where $\Delta_{c}=\frac{2 G_{c}}{\sigma_{c}}$ is critical opening for a linear cohesive law (see Fig. 7), and where \pm sign depends on the direction of bending. Let us remark that in relation (71), sign + is used if $\tilde{m}_{0}^{22}<0$, while sign - is used otherwise.

\subsection{Mode $I I$}

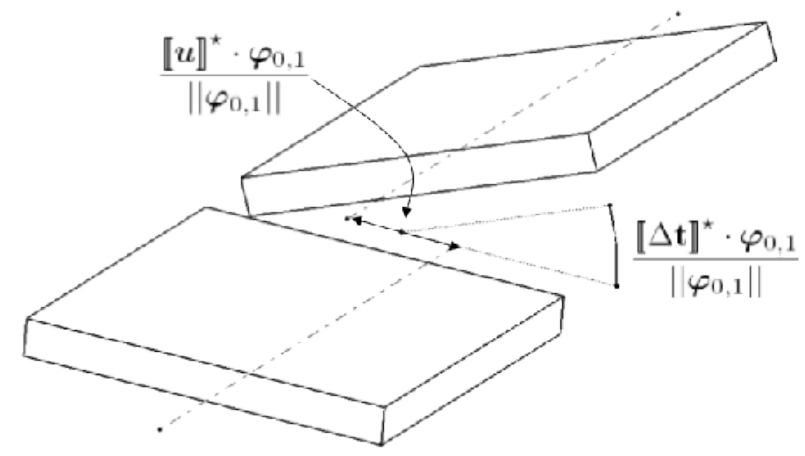

Figure 8: The two components of the tangential opening $\Delta_{t}^{\star}$. Before opening, the element axis are the same and the two crack lips are in the same plane.

Following exactly the same lines for the tangential effective opening in mode II drawn on figure 8,

$$
\Delta_{t}^{\star}=\left(1-\eta_{I I}\right) \frac{\llbracket \boldsymbol{u} \rrbracket^{\star} \cdot \varphi_{0,1}}{\left\|\varphi_{0,1}\right\|} \pm \eta_{I I} \frac{h}{6} \frac{\llbracket \Delta \boldsymbol{t} \rrbracket^{\star} \cdot \boldsymbol{\varphi}_{0,1}}{\left\|\boldsymbol{\varphi}_{0,1}\right\|} .
$$

In this expression, the parameter $\eta_{I I}$ ensures that the coupling between the resulting shear stress and the resulting torsion respects the energetic balance, which is obtained for

$$
\eta_{I I}=\frac{\frac{6}{h}\left|\left\langle\tilde{m}_{0}^{21}\right\rangle\right|}{\left\langle n_{0}^{21}\right\rangle+\frac{6}{h}\left|\left\langle\tilde{m}_{0}^{21}\right\rangle\right|}
$$

where $n_{0}^{21}$ and $\tilde{m}_{0}^{21}$ are respectively the shearing effort and the torsion torque at fracture initialization. Finally, sign + is used in Eq. (78) when $\tilde{m}_{0}^{21}<0$, and otherwise the sign - is used, following the same convention given for $\tilde{m}_{0}^{22}$ in mode $\mathrm{I}$. 


\subsection{Mode Combination}

Now the combination between mode I and II is realized in a similar way as achieved by several authors [7, 16, 47-51] when considering Cauchy stress tensors for 3D TSL. This method, which was first suggested by Camacho et al. [3], and extended a few years later by Ortiz et al. [46], considers an effective stress $\sigma_{\text {eff }}$ to detect fracture initialization, with the criteria: $\sigma_{\text {eff }}>\sigma_{\mathrm{c}}$, and allows for fracture in compression to happen if the shearing stress is sufficiently large,

$$
\sigma_{\mathrm{eff}}=\left\{\begin{array}{ll}
\sqrt{\sigma^{2}+\beta^{-2} \tau^{2}} & \text { if } \sigma \geq 0 \\
\frac{1}{\beta} \ll|\tau|-\mu|\sigma| \gg & \text { if } \sigma<0
\end{array} .\right.
$$

In this criterion, $\sigma$ and $\tau$ are respectively the normal and tangential Cauchy stress at the integration point where fracture is evaluated, $\beta=\frac{K_{I I c}}{K_{I c}}$ is the coupling mode parameter, and $\mu$ is the friction parameter, these two last parameters depending on the material only. The operator $\ll \bullet \gg$ is equal to $\bullet$ if $\bullet \geq 0$ and 0 otherwise. The initiation criterion (80) can still be considered in the present work. Indeed, from the resulting stresses of the shell formulation, the Cauchy stress tensor can be directly evaluated through the thickness of the body, either analytically for linear elasticity or at Simpson points on the thickness for non-linear shells.

However, in this new formulation for thin structures the cohesive law should be written in terms of resulting values instead of Cauchy stress, and some quantities and notations have to be first introduced:

- The effective opening $\Delta^{\star}$ is a combination of the two effective openings $\Delta_{n}^{\star}$ (71) and $\Delta_{t}^{\star}$ (78). So $\Delta^{\star}$ allows to take into account a coupling between the two fracture mode. Its value is an extension of the formulation, presented by Ortiz et al in [46], of thin structures:

$$
\Delta^{\star}=\sqrt{\ll \Delta_{n}^{\star} \gg^{2}+\beta^{2} \Delta_{t}^{\star 2}} .
$$

The use of the operator $\ll \bullet \gg$ is mandatory. Indeed if the rupture occurs in compression, the normal opening has to be equal to zero, as in compression the normal opening is negative, which means that there is a penetration between element. Obviously this latter case has no physical meaning and forces have to be introduced between elements. In place of contact forces, the DG terms $a_{n I 2}^{s}\left(\boldsymbol{\varphi}_{h}, \delta \boldsymbol{\varphi}\right)$ and $a_{n I 3}^{s}\left(\boldsymbol{\varphi}_{h}, \delta \boldsymbol{\varphi}\right)$ (see equations (51) and (55)) can also be used to weakly enforce a zero penetration.

- The critical opening $\Delta_{c}$ is the opening for which the fracture process is completed, meaning no remaining forces exist between the fractured sides. Therefore, for this value, the energy released has to be equal to $G_{c}$, and for linear decreasing monotonic cohesive laws, $\Delta_{c}=\frac{2 G_{c}}{\sigma_{c}}$.

- The maximal effective opening reached during the simulation $\Delta_{\max }^{\star}$, is an internal variable tracking opening history. 
Now the cohesive law can be rewritten in terms of these new definitions. As it is well known that for brittle materials the shape of the cohesive law has little influence on numerical results, as long as the law is monotonically decreasing, a simple linear decreasing law is considered in this work. In case of unloading the effort decreases linearly to zero (see Fig. 7). By application of cohesive principle on stress resultant vectors, the following cohesive model is found:

1. Tensile case $(\sigma \geq 0 \text { at mid-surface })^{8}$,

- if $\Delta^{\star} \geq \Delta_{\text {max }}^{\star}$ (loading case),

$$
\begin{aligned}
\left\langle\tilde{m}^{22}\right\rangle_{\mathrm{coh}} & =\tilde{m}_{0}^{22}\left(1-\frac{\Delta^{\star}}{\Delta_{c}}\right) \frac{\Delta_{n}^{\star}}{\Delta^{\star}} \\
\left\langle n^{22}\right\rangle_{\mathrm{coh}} & =n_{0}^{22}\left(1-\frac{\Delta^{\star}}{\Delta_{c}}\right) \frac{\Delta_{n}^{\star}}{\Delta^{\star}} \\
\left\langle\tilde{m}^{21}\right\rangle_{\mathrm{coh}} & =\tilde{m}_{0}^{21} \beta\left(1-\frac{\Delta^{\star}}{\Delta_{c}}\right) \frac{\left|\Delta_{t}^{\star}\right|}{\Delta^{\star}} \\
\left\langle n^{21}\right\rangle_{\mathrm{coh}} & =n_{0}^{21} \beta\left(1-\frac{\Delta^{\star}}{\Delta_{c}}\right) \frac{\left|\Delta_{t}^{\star}\right|}{\Delta^{\star}}
\end{aligned}
$$

- if $\Delta^{\star}<\Delta_{\text {max }}^{\star}$ (unloading case),

$$
\begin{aligned}
\left\langle\tilde{m}^{22}\right\rangle_{\mathrm{coh}} & =\tilde{m}_{0}^{22}\left(\frac{\Delta^{\star}}{\Delta_{\text {max }}^{\star}}-\frac{\Delta^{\star}}{\Delta_{c}}\right) \frac{\Delta_{n}^{\star}}{\Delta^{\star}} \\
\left\langle n^{22}\right\rangle_{\mathrm{coh}} & =n_{0}^{22}\left(\frac{\Delta^{\star}}{\Delta_{\text {max }}^{\star}}-\frac{\Delta^{\star}}{\Delta_{c}}\right) \frac{\Delta_{n}^{\star}}{\Delta^{\star}} \\
\left\langle\tilde{m}^{21}\right\rangle_{\mathrm{coh}} & =\tilde{m}_{0}^{21} \beta\left(\frac{\Delta^{\star}}{\Delta_{\text {max }}^{\star}}-\frac{\Delta^{\star}}{\Delta_{c}}\right) \frac{\left|\Delta_{t}^{\star}\right|}{\Delta^{\star}} \\
\left\langle n^{21}\right\rangle_{\mathrm{coh}} & =n_{0}^{21} \beta\left(\frac{\Delta^{\star}}{\Delta_{\text {max }}^{\star}}-\frac{\Delta^{\star}}{\Delta_{c}}\right) \frac{\left|\Delta_{t}^{\star}\right|}{\Delta^{\star}}
\end{aligned}
$$

2. Compression case ( $\sigma<0$ at mid-surface),

- if $\Delta^{\star} \geq \Delta_{\text {max }}^{\star}$ (loading case),

$$
\begin{aligned}
& \left\langle\tilde{m}^{21}\right\rangle_{\mathrm{coh}}=\tilde{m}_{0}^{21}\left(1-\frac{\Delta^{\star}}{\Delta_{c}}\right) \\
& \left\langle n^{21}\right\rangle_{\mathrm{coh}}=n_{0}^{21}\left(1-\frac{\Delta^{\star}}{\Delta_{c}}\right)
\end{aligned}
$$

- if $\Delta^{\star}<\Delta_{\max }^{\star}$ (unloading case),

$$
\begin{aligned}
\left\langle\tilde{m}^{21}\right\rangle_{\mathrm{coh}} & =\tilde{m}_{0}^{21}\left(\frac{\Delta^{\star}}{\Delta_{\max }^{\star}}-\frac{\Delta^{\star}}{\Delta_{c}}\right) \\
\left\langle n^{21}\right\rangle_{\mathrm{coh}} & =n_{0}^{21}\left(\frac{\Delta^{\star}}{\Delta_{\max }^{\star}}-\frac{\Delta^{\star}}{\Delta_{c}}\right)
\end{aligned}
$$

\footnotetext{
${ }^{8}$ Note that the cohesive zone is in term of the traction components $n^{\alpha \beta}$ and not $\tilde{n}^{\alpha \beta}$
} 
where in this last case the condition $\frac{\left|\Delta_{t}^{\star}\right|}{\Delta^{\star}}=\frac{1}{\beta}(c f$. equation (81)) is taken into account.

The use of $n_{0}^{22}, \tilde{m}_{0}^{22}, n_{0}^{21}, \tilde{m}_{0}^{21}$ allows to guarantee the continuity of stress at fracture initialization. If this continuity is not ensured, K.D Papoulia et al [50] have demonstrated that there are some convergence problems. Furthermore, as at fracture initialization the ratios $\frac{\Delta_{n}^{\star}}{\Delta^{\star}}$ and $\frac{\left|\Delta_{t}^{\star}\right|}{\Delta^{\star}}$ are undetermined, their initial values are chosen respectively equal to 1 and $\frac{1}{\beta}$ in order to ensure the continuity of efforts. It has to be noticed that the choice of tensile or compressive case is performed at fracture initialization. Therefore, although unloading and/or compression/tension shifts can be accounted for during the fracture process, as shown on Fig. 7, this model is not enable to shift from a fracture process in tension to a fracture process in compression (e.g. start the fracture in compression and end up the fracture in tension). However the model could be enhanced. Finally, with these definitions, the cohesive terms $a_{\text {cohesive }}^{s}\left(\llbracket \varphi_{h} \rrbracket, \llbracket \delta \varphi \rrbracket\right)$ of equation (68) can be written

$$
\begin{aligned}
a_{\text {cohesive }}^{s}\left(\llbracket \boldsymbol{\varphi}_{h} \rrbracket, \llbracket \delta \boldsymbol{\varphi} \rrbracket\right)= & \int_{s}\left\langle\boldsymbol{n}^{\alpha}\right\rangle_{\mathrm{coh}} \cdot \llbracket \delta \boldsymbol{\varphi} \rrbracket \nu_{\alpha}^{-} d \partial \mathcal{A}_{e} \\
& +\int_{s}\left\langle\tilde{\boldsymbol{m}}^{\alpha}\right\rangle_{\mathrm{coh}} \cdot \llbracket \delta \Delta \boldsymbol{t} \rrbracket \nu_{\alpha}^{-} d \partial \mathcal{A}_{e},
\end{aligned}
$$

where the component of $\left\langle\boldsymbol{n}^{\alpha}\right\rangle_{\text {coh }}$ and $\left\langle\tilde{\boldsymbol{m}}^{\alpha}\right\rangle_{\text {coh }}$ are computed thanks to the set of equations $(82-93)$, with others components of the resultant stresses equal to zero. Note that all these definitions depend on the choice of manifold used on the interface. As mentioned earlier, this paper follows this convention: $\varphi_{0,1}$ is parallel to the interface and $\varphi_{0,2}$ is perpendicular to interface, as it is illustrated on Fig. 5.

\section{Implementation}

All developments are implemented in $\mathrm{C}++$ with the integration of solvers to Gmsh [52], which is also used for pre-post operations. Furthermore, the objects of Gmsh are linked to the solvers thanks to a library in such a way that all classes defined in Gmsh can be used in the solver. The main feature of the dof manager implemented in Gmsh is its ability to create dofs independently of the nodes. In fact, for a full-DG implementation, it is possible to create many dofs on the same node of a mesh. Therefore, instead of duplicating nodes, as usually done for DG formulations, 3 displacement dofs are created for each element linked to a vertex. Proceeding this way avoids mesh and topology modifications when importing or creating a finite element mesh.

Two solvers are implemented: on the one hand a quasi-static solver using Taucs [53, 54] or PETSc [55-57] to solve a system $\mathbf{K q}=\mathbf{g}$, with $\mathbf{K}$ the stiffness matrix, $\mathbf{q}$ the unknowns vector and $\mathbf{g}$ the external force vector. On the other hand, a dynamic explicit solver based on the $\alpha$-generalized method [58] is implemented, which allows introducing numerical damping in the problem. This 
last solver uses PETSc vector to store all data in such a way that the resolution algorithms can be very easily implemented on a vectorized form using the efficient PETSc vector operations.

The elementary stiffness matrix and elementary force vector are implemented following the $C^{0} / \mathrm{DG}$ formulation described in [34], in which the new terms related to the full DG formulation, i.e. $a_{n I}^{s}\left(\boldsymbol{\varphi}_{h}, \delta \boldsymbol{\varphi}\right)$ and $a_{s I}^{s}\left(\boldsymbol{\varphi}_{h}, \delta \boldsymbol{\varphi}\right)$ can be obtained easily with the same argumentation. In particular, the DG implementation is locking-free, when considering under-integrated quadratic elements or fully integrated cubic elements. The library of elements implemented is

- 6-node quadratic triangles with 3 Gauss points for bulk integration and 2 Gauss points on each edge for interface integration;

- 9-node quadratic quadrangles with 4 Gauss points for bulk integration and 3 Gauss points on each edge for interface integration;

- 10-node cubic triangles with 6 Gauss points for bulk integration and 4 Gauss points on each edge for interface integration;

- 16-node quadratic quadrangles with 16 Gauss points for bulk integration and 4 Gauss points on each edge for interface integration.

As the DG method results in a one field formulation [34], each element has three (displacement) dofs per node.

\section{Numerical applications}

Some numerical applications are now performed to demonstrate the ability of the presented framework to simulate fracture problems. The first application models an in-plane pure mode I crack propagation, the second one investigates an out-of-plane through-the-thickness crack propagation and the last one studies the effect of a blast on a cracked cylinder. The obtained results are compared to literature data or to analytical results. Note that for all performed simulations the values of stability parameters are: $\beta_{1}=\beta_{2}=\beta_{3}\left(\frac{L_{c}}{h}\right)^{2}=10$, with $h$ the thickness and $L_{c}$ the largest dimension of the problem. Although it has been seen in section 3.4 that $\beta_{1}=10$ is close to the stability threshold, this choice is motivated by the stable results obtained for finer meshes. Furthermore, a reduced value is recommended for explicit dynamic problems as the critical time step is proportional to $\frac{1}{\sqrt{\max \beta_{i}}}[39]$.

\subsection{Mode I dynamic crack propagation: spall test of a notched specimen}

This example, performed by Zavattieri [59], considers the dynamic crack propagation of the single-edge notched specimen represented on Fig. 9. The different material properties are summarized in Table 2. Contrarily to [59], where cohesive elements are only inserted along a predefined crack path, with the full-DG framework the crack can a priori follows any direction. As the crack 


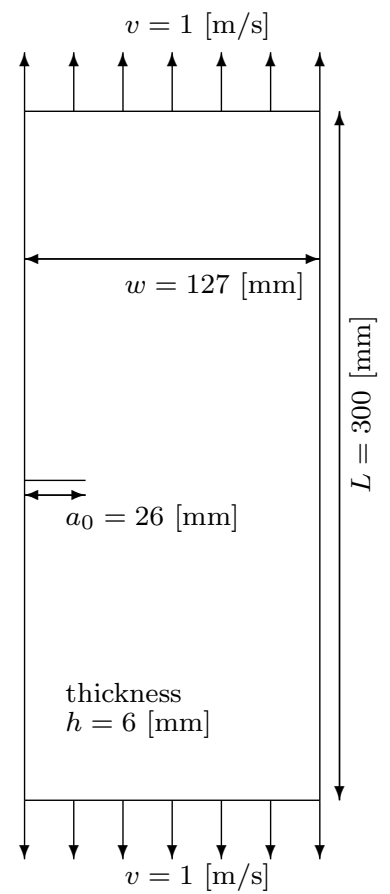

Figure 9: Single-edge notched specimen.

should propagate straightforwardly, for symmetry reasons, the test is numerically performed using structured and unstructured meshes successively in order to show the convergence of the method for both mesh configurations. Results on a structured mesh of $3200=40 \times 80$ (width $\times$ length) bi-cubic quadrangles are considered as benchmark.

The test is performed using an explicit time integration algorithm without numerical dissipation (spectral radius equal to one). The force vs. displacement curve at the top edge of the plate is illustrated on Fig. 11 a), where some characteristic points obtained by Zavattieri [59] are also reported, showing an excellent correlation between the results. This force vs. displacement curve shows the effect of a wave propagation in the bar, reflecting on the symmetry axis, and leading to crack propagation. Figure $11 \mathrm{~b}$ ) plots the energy released during the simulation:

$$
G=\frac{W_{e x t}-W_{i n t}}{h \Delta a},
$$

where $W_{\text {ext }}$ is the work of external forces, $W_{\text {int }}$ is the work of internal forces, $h$ is the thickness and $\Delta a$ is the final increment of crack length $(101[\mathrm{~mm}]$ in this case). The graphs shows that $G$ increases (linearly) only during crack propagation and stabilizes itself at $12.021\left[\mathrm{~kJ} / \mathrm{m}^{2}\right]$ which is close to the material fracture energy of $12.250\left[\mathrm{~kJ} / \mathrm{m}^{2}\right]$ within a $1.8 \%$ relative error. It is believed 


\begin{tabular}{ll}
\hline Property & Value \\
\hline Young modulus $[\mathrm{GPa}]$ & 200 \\
Poisson ratio $[-]$ & 0.3 \\
Density $\left[\mathrm{kg} / \mathrm{m}^{3}\right]$ & 7850 \\
Fracture energy $\left[\mathrm{J} / \mathrm{m}^{2}\right]$ & 12250 \\
Fracture stress $[\mathrm{MPa}]$ & 700 \\
Coupling parameter $\beta[-]$ & 1 \\
Frictional coefficient $[-]$ & 0. \\
\hline
\end{tabular}

Table 2: Material properties for the single-edge notched test.

that this small error results from the temporal discretization which introduces a truncating positive or negative error on the cohesive law, as illustrated on Fig. 10. Indeed when computing the work of external forces, and thus the dissipated energy, only the archived values (markers "+" on Fig. 10) are considered, leading to an error vanishing when the time step size decreases. This test

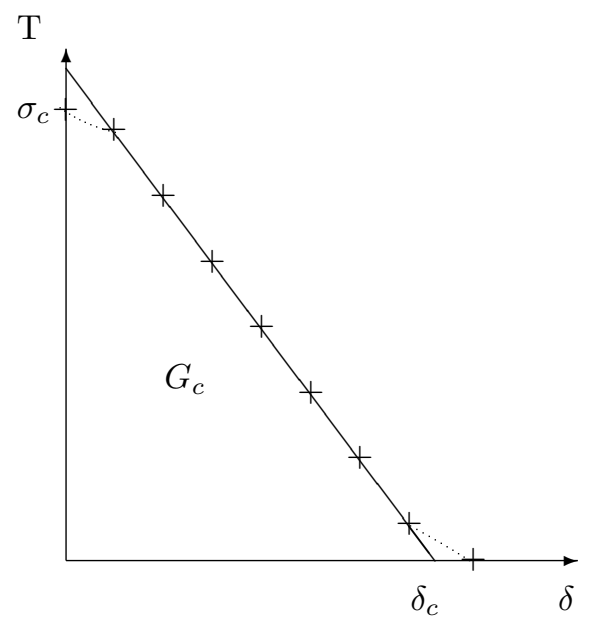

Figure 10: Truncation error on the cohesive law due to time discretization, represented with markers "+".

demonstrated the ability of the presented fracture model to dissipate the correct amount of energy during the fracture process, under dynamical experiments.

Furthermore, this example is also simulated with two unsymmetric unstructured meshes. These two meshes are built with quadratic 6-node triangles, which are generated by prescribing a distance between two nodes at the crack tip of 2.5 and $5[\mathrm{~mm}]$ successively. Further from the crack, the element sizes is twice larger. These specifications produce a coarse mesh of approximately 900 elements and a fine mesh of 2600 elements. Two simulations are performed with 


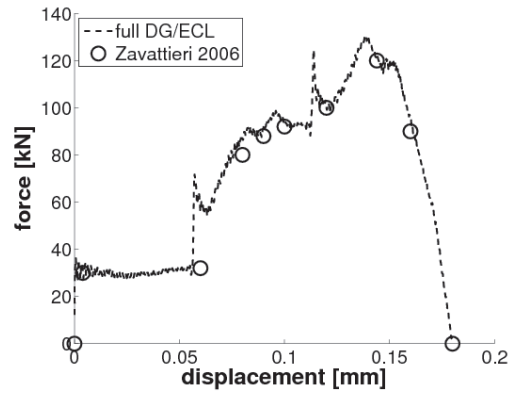

(a)

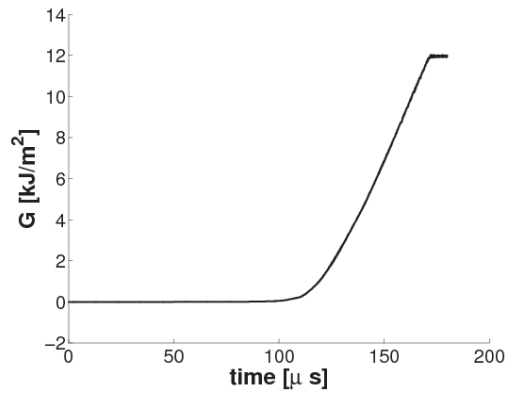

(b)

Figure 11: Results of single-edge notched specimen for a structured mesh. a) Forcedisplacement relation. b) Released energy.

the coarse mesh, one without numerical dissipation and the other one with a low numerical damping (spectral radius of 0.9). Crack paths obtained with these unstructured meshes are illustrated on Fig. 13. It can be seen, that for the coarse mesh, a crack bifurcation appears if no numerical damping is introduced Fig. 13 a), while the use of numerical damping prevents this behavior, Fig. 13 b). The crack path obtained with the finer mesh and a spectral radius of 0.9 is illustrated on Fig. 13 c). In the three cases some elements blew up during the simulation. This phenomenon is purely numeric and occurs due to the combination of the fracture criterion and of the Gauss integration performed on the interface. The fracture is introduced at a Gauss point when a effective stress is larger than a characteristic material stress (cf. Eq. (80)). Thus, the criterion can be satisfied at two Gauss points of adjacent edges at the same time step (see Fig. 12 for quadratic triangles). This is particularly true for small elements as the integration points on two different edges can be close. This eventually can lead to the blow up of an element. One way of avoiding this is to reduce the time step, or to give a statistical distribution of critical stress for the interface elements [60].

Results are in agreement, as it is also observed on Fig. 14, which plots the force vs. displacement relation obtained in the different cases. All curves are similar, which demonstrated that the unstructured meshes predict an excellent global result even if they don't reproduce the exact crack path ${ }^{9}$. In particular, the finer mesh, which has the worst reproduction of the crack path, shows a relative error of $2.7 \%$ on maximal force, and of $4.3 \%$ on the time where the force vanishes to zero. 


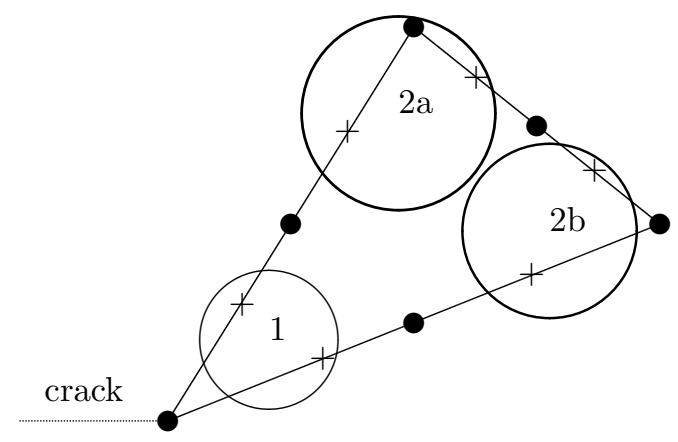

Figure 12: Activation of fracture at two Gauss points $(+)$ on different edges. The initiation can occur simultaneously at both Gauss points of group 1 if the rupture criterion is reached at both points. The criterion can also be met later on for groups $2 \mathrm{a}$ and $2 \mathrm{~b}$ independently. If all integration points are broken the element can blow up.

\subsection{Out-of-plane crack propagation: Double clamped plate in bending}

The through-the-thickness crack propagation is studied with the test presented by the authors in [30] for beams. It consists of a plate, clamped at its two extremities, for which the vertical displacement $\left(u_{z}\right)$ at its middle $(c f$. Fig. $15)$ is prescribed in a quasi-static way. Thus, the symmetry axis of the plate sees the larger stress at its skin. A crack initiates at the bottom part (under tension), and should propagate through the thickness of the plate. Although we have a shell model, such a propagation is possible considering the cohesive law described in section 4 . Furthermore, a pre-tension or a pre-compression can be applied as initial boundary conditions by prescribing a small displacement $\left(u_{x}\right)$ along the plate axis. This initial value modifies the critical $u_{z}$ leading to crack initiation, and can also modify the fracture stability. Indeed, as it is explained in details in [30], the difference of internal energy between the un-fractured configuration (one double clamped plate of length $L_{D C P}$ ) and the fractured configuration (two simply clamped plates of length $L_{D C P} / 2$ ) can be determined numerically at fracture initialization from

$$
\Delta E_{\text {int }}=\frac{h L_{D C P} w}{2 E}\left(\frac{13}{12} \eta_{I}^{2}-2 \eta_{I}+1\right) \sigma_{\mathrm{c}}^{2}
$$

\footnotetext{
${ }^{9}$ Experimentally due to some defects in the material an uncertainty also exists on the crack path.
} 


\begin{tabular}{ll}
\hline Material property & Value \\
\hline Young modulus [GPa] & 71 \\
Poisson ratio [-] & 0. \\
Fracture energy $\left[\mathrm{J} / \mathrm{m}^{2}\right]$ & 8800 \\
Fracture stress $[\mathrm{MPa}]$ & 400 \\
Coupling parameter $\beta[-]$ & 1 \\
Frictional coefficient [-] & 0. \\
\hline
\end{tabular}

Table 3: Material properties for the double clamped plate test.

where in this formula, $E$ and $\sigma_{\mathrm{c}}$ are respectively the Young modulus and the fracture stress ( $c f$. Table 3 for material properties) and $\eta_{I}$ is the coupling parameter depending on the pre-tension, Eq. (74). If this difference of energy is larger than the fracture energy, the fracture occurs in one increment of the vertical displacement, otherwise the vertical displacement has to keep increasing in order to propagate the crack through the plate's thickness. Thus, the fracture process is stable for $\eta_{I}$ between the roots of equation $\Delta E_{\text {int }}=h G_{c} w$, which gives for the present case $0.6678<\eta_{I}<1.1783$. In this test, $u_{x}$ ranges from -0.02 $\mathrm{mm}$ to $1 \mathrm{~mm}$, by increments of $0.02 \mathrm{~mm}$, leading to stable through-the-thickness crack propagation, except in the case $u_{x}=0.1[\mathrm{~mm}]$ for which $\eta_{I}=0.655$.

The graphs a) and b) of Fig. 16 focus on the case without pre-tension $u_{x}=0$. This test is performed with three different meshes:

1. A structured mesh generated with bi-cubic quadrangles. This mesh comprises $5 \times 40$ (width $\times$ length) elements;

2. An unstructured mesh generated with quadratic triangles, which the characteristic length is $1[\mathrm{~mm}]$. This mesh comprises approximately 860 elements;

3. An unstructured mesh generated with cubic triangles which the characteristic length is $1[\mathrm{~mm}]$. This mesh comprises approximately 860 elements.

Fig. 16 a) shows the resulting force vs. displacement curve, which is also evaluated analytically for the initial and final configurations. The obtained numerical results are very close to the analytical solution for all meshes, and the transition between the un-fractured and fractured states models the through-the-thickness crack propagation. During this fracture process the released energy (95) is depicted on graph b). Once again, this energy is very close to the analytical value of $8800\left[\mathrm{~J} / \mathrm{m}^{2}\right]$, with a relative error of $0.1 \%$ only. The same test is now performed with the structured mesh for different pre-tensions, and the resulting force vs. displacement curve is reported on Fig. $16 \mathrm{c}$ ). In agreement with the theory, the crack propagation is stable, except in the case $u_{x}=0.1[\mathrm{~mm}]$, for which rupture happens in a single displacement increment. This test allows concluding to the ability of the suggested model to propagate a crack through the thickness of the shell. 


\begin{tabular}{ll}
\hline Property & Value \\
\hline Height $[\mathrm{m}]$ & 1 \\
Diameter $[\mathrm{m}]$ & 1.2 \\
Thickness $[\mathrm{mm}]$ & 1 \\
Young modulus [GPa] & 73.1 \\
Poisson ratio [-] & 0.33 \\
Density $\left[\mathrm{kg} / \mathrm{m}^{3}\right]$ & 2780 \\
Fracture energy [J/m $\left.{ }^{2}\right]$ & 67000 \\
Fracture stress $[\mathrm{MPa}]$ & 642 \\
Coupling parameter $\beta[-]$ & 1 \\
Frictional coefficient $[-]$ & 0. \\
\hline
\end{tabular}

Table 4: Geometrical \& material properties for the cylinder test.

\subsection{Blast of cracked cylinder}

This last example, presented by R. Larsson et al [24], consists of a blast wave interacting with a cracked cylinder initially pressurized at 2 [bars]. The 56-mm-long crack is parallel to the cylinder axis and is centered on its height. Geometrical and material properties of the cylinder are summarized in Table 4. The blast is simulated by applying a pressure, on the cylinder inner wall, described by Fig. 17, while top and the bottom faces are clamped.

This test is simulated using an explicit time-stepping scheme ${ }^{10}$ with a spectral radius equals to 1 . Three meshes are considered:

1. a structured mesh of $720=60 \times 12$ (diameter $\times$ height) bi-cubic quadrangles. The elements edge is approximately 4 -cm long;

2. a structured mesh of $2880=120 \times 24$ (diameter $\times$ height) bi-cubic quadrangles. The elements edge is approximately 2 -cm long;

3. an unstructured mesh with a characteristic mesh sizes of $2[\mathrm{~cm}]$ near the crack tip such a way that it contains approximately 1200 bi-quadratic triangles.

For this test the symmetry is taken into account to reduce the time of simulations, and only the upper part of the cylinder is modeled. Fig. 19 and 20 show snapshots of the simulation to illustrate the crack propagation which is almost the same for all meshes. The final crack path draws a funnel shape which is more visible for the unstructured mesh due to its ability to propagate crack in many directions. Indeed, with the structured mesh the crack can propagate only in two directions: straightforward and at 90-degree angles, which facilitates a straight propagation. Despite that, the funnel shape is still appearing at the end.

This benchmark has recently been performed by R. Larsson et al [24], where a cohesive zone model accounting for viscosity is used. As this last parameter

${ }^{10}$ The pressure is initialized to 2 [bars] using a quasi-static scheme 
is not present in the presented model some differences appear in the results, in particular the crack propagation speed is higher than in [24], as shown in Fig. 18. The curves of both structured meshes are close to each other, which shows the convergence of results. For the unstructured mesh the curve oscillates around these curves, showing the average speed is well captured, but due to the unstructured nature of the mesh, the inter-element openings is not regular in time.

It has to be noticed that as other cracks are initiated at the cylinder extremities, we can only study the crack propagation on a distance of about $0.33[\mathrm{~m}]$ to compare with the results of [24], in which new cracks cannot be initiated. This phenomenon appears clearly on Fig. 19, where it is shown that the central crack propagates between approximately $99[\mu \mathrm{s}]$ and $189[\mu \mathrm{s}]$, after which the pressure becomes too high at at the cylinder's top, resulting into the initiation of new cracks. Afterward cracks propagate from both sides and join together at $226[\mu \mathrm{s}]$. Note that during this last stage several cracks appear in the whole cylinder.

\section{Conclusion}

This paper focuses on the extension to shell formulation of the framework combining a full DG formulation and the extrinsic cohesive law.

First it is proved that the suggested full DG formulation is able to simulate continuum shell mechanics. Moreover, it is set out that the numerical properties of consistency and convergence rate are optimal. Obviously, the main interest of this presented framework is its ability to simulate fracture initiation and propagation using the interface elements, in order, either, to integrate the DG terms (before fracture), or to integrate the cohesive law once a fracture criterion is reached.

Then, in order to be able to simulate a through-the-thickness crack propagation, a new cohesive law is defined from the resulting stress vectors inherent to the shell formulations.

Finally, the capacity of the proposed framework is demonstrated from several benchmarks for which the solutions are found to be close to the ones presented in the literature.

Nevertheless, the presented cohesive law is valid for perfectly brittle materials only, and has to be improved in further work to simulate crack propagation for more complex (ductile) material behaviors.

\section{Appendix A. Demonstration of numerical properties}

The proposed full-DG framework respects consistency and stability, while having the optimal convergence rate. 


\section{Consistency}

Following the same token given by authors in [30], the consistency is proved by considering the exact solution $\boldsymbol{u}$. This exact solution is $\mathcal{C}^{2}(\mathcal{S})$, which implies $\llbracket \boldsymbol{\Phi} \rrbracket=0$ (i.e. $\llbracket \boldsymbol{\varphi} \rrbracket=\llbracket \Delta \boldsymbol{t} \rrbracket=0)$ in $\mathcal{S}$ and the relation (61) becomes,

$$
\begin{gathered}
\sum_{e} \int_{\mathcal{A}_{e}}\left(\bar{\rho} \ddot{\boldsymbol{\varphi}} \cdot \delta \boldsymbol{\varphi}-\bar{j}_{0} \boldsymbol{n}^{\alpha}(\boldsymbol{\varphi}) \cdot \delta(\boldsymbol{\varphi})_{, \alpha}-\bar{j}_{0} \tilde{\boldsymbol{m}}^{\alpha}(\boldsymbol{\varphi}) \cdot \delta \Delta \boldsymbol{t}_{, \alpha}\right) d \mathcal{A} \\
-\sum_{s} \int_{s}\left(\left\langle\bar{j}_{0} \boldsymbol{n}^{\alpha}\right\rangle \cdot \llbracket \delta \boldsymbol{\varphi} \rrbracket \nu_{\alpha}^{-} d \partial \mathcal{A}_{e}+\left\langle\bar{j}_{0} \tilde{\boldsymbol{m}}^{\alpha}\right\rangle \cdot \llbracket \delta \Delta \boldsymbol{t} \rrbracket \nu_{\alpha}^{-} d \partial \mathcal{A}_{e}\right)=0,
\end{gathered}
$$

As for the exact solution $\boldsymbol{n}^{\alpha}$ and $\tilde{\boldsymbol{m}}^{\alpha}$ are continuous, the last relation becomes, after integrating by parts on each element,

$$
\begin{aligned}
0= & \int_{\mathcal{A}} \bar{\rho} \ddot{\boldsymbol{\varphi}} \cdot \delta \boldsymbol{\varphi} d V-\int_{\mathcal{A}} \bar{j}_{0}\left(\bar{j} \boldsymbol{n}^{\alpha}\right)_{, \alpha} \cdot \delta \boldsymbol{\varphi} d \mathcal{A} \\
& -\int_{\mathcal{A}}\left(\bar{j} \tilde{\boldsymbol{m}}^{\alpha}\right)_{, \alpha} \cdot \delta \Delta \boldsymbol{t} d \mathcal{A}
\end{aligned}
$$

which implies due to the arbitrary nature of test functions,

$$
\begin{array}{ll}
\bar{\rho} \ddot{\boldsymbol{\varphi}}-\frac{1}{\bar{j}}\left(\bar{j} \boldsymbol{n}^{\alpha}\right)_{, \alpha}=0 & \text { on } \mathcal{A}, \text { and } \\
\overline{\bar{j}}\left(\bar{j} \tilde{\boldsymbol{m}}^{\alpha}\right)_{, \alpha}+\lambda \boldsymbol{t}=0 & \text { on } \mathcal{A} .
\end{array}
$$

These two relations are identical to the strong formulation of the problem described by the equations (2) and (3) where the shearing is neglected $(\boldsymbol{l}=0)$, which proves the consistency of the formulation.

Stability

The stability can be demonstrated by combining the developments given in $[30,34]$. If by simplicity, the prescribed displacement $\overline{\boldsymbol{u}}$ and direction $\overline{\boldsymbol{\Delta}} \boldsymbol{t}$ are taken to 0 and that there is no inertial forces, the following energetic norm can 
be suggested,

$$
\begin{aligned}
& \left.\|\boldsymbol{u}\|\right|^{2}=\sum_{e}\left\|{\sqrt{\mathcal{H}_{n} \bar{j}_{0}}}^{\alpha \beta} \frac{1}{2}\left(\boldsymbol{\varphi}_{0, \alpha} \cdot \boldsymbol{u}_{, \beta}+\boldsymbol{u}_{, \alpha} \cdot \boldsymbol{\varphi}_{0, \beta}\right)\right\|_{L^{2}\left(\mathcal{A}_{e}\right)}^{2}+ \\
& \sum_{e}\left\|{\sqrt{\mathcal{H}_{m} \bar{j}_{0}}}^{\alpha \beta}\left(\boldsymbol{\varphi}_{0, \alpha} \cdot \boldsymbol{\Delta} \boldsymbol{t}_{, \beta}+\boldsymbol{u}_{, \alpha} \cdot \boldsymbol{t}_{0, \beta}\right)\right\|_{L^{2}\left(\mathcal{A}_{e}\right)}^{2}+ \\
& \sum_{s}\left\|\sqrt{\frac{\beta_{1} \mathcal{H}_{m} \bar{j}_{0}}{h_{s}}} \varphi_{0, \alpha} \cdot \llbracket \Delta t \rrbracket \nu_{\beta}^{-}\right\|_{L^{2}(s)}^{2}+ \\
& \sum_{s}\left\|{\sqrt{\frac{\beta_{2} \mathcal{H}_{n} \bar{j}_{0}}{h_{s}}}}^{\alpha \beta} \varphi_{0, \alpha} \cdot \llbracket \boldsymbol{u} \rrbracket \nu_{\beta}^{-}\right\|_{L^{2}(s)}^{2}+
\end{aligned}
$$

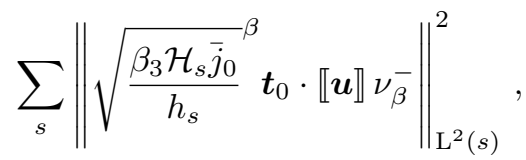

with the abuses of notation

$$
\begin{aligned}
& \left\|\sqrt{\mathcal{H}}^{\alpha \beta} a_{\alpha \beta}\right\|_{L^{2}\left(\mathcal{A}_{e}\right)}^{2}=\int_{\mathcal{A}_{e}} a_{\alpha \beta} \mathcal{H}^{\alpha \beta \gamma \delta} a_{\gamma \delta} d \mathcal{A}, \text { and } \\
& \sum_{s}\left\|\sqrt{\mathcal{H}}^{\alpha \beta} \llbracket a_{\alpha} \rrbracket \nu_{\beta}^{-}\right\|_{L^{2}(s)}^{2}=\sum_{e} \frac{1}{2} \int_{\partial \mathcal{A}_{e}} \mathcal{H}^{\alpha \beta \gamma \delta} a_{\alpha} \nu_{\beta} a_{\gamma} \nu_{\delta} d \partial \mathcal{A}_{e}
\end{aligned}
$$

In Eq. (A.7), integration on all sides $s$ is equivalent to one half of the integration on all the element boundaries. Indeed, the sum on all the element boundaries accounts twice for a side $s^{11}$.

Expression (A.5) is a norm, i.e. its value is equal to zero only for $\boldsymbol{u}=0$ on $\mathcal{A}_{h}$. Indeed, if \|\| $\boldsymbol{u} \| \mid$ is equal to zero, then all the contributions are also equal to zero but, if this is the case, the only solution is $\boldsymbol{u}=0$ on $\mathcal{A}_{h}$, as it is shown in the following lines. If the membrane energy (first term of Eq. (A.5)) is equal to zero, then the solution of the problem is $\boldsymbol{u}_{\alpha}$ parallel to $\boldsymbol{t}_{0}$ on every $\mathcal{A}_{e}$. If the bending term (second term of Eq. (A.5)) is equal to zero, it means that $\rho_{\alpha \beta}=0$ on $\mathcal{A}_{e}$. Using Eq. (12), and since $\boldsymbol{u}_{, \alpha}$ is parallel to $\boldsymbol{t}_{0}$, the solution $\rho_{\alpha \beta}=0$ implies $C=\boldsymbol{u}_{, \alpha} \cdot \boldsymbol{t}_{0}$ is constant on each $\mathcal{A}_{e}$. Since the jump in the variation $\Delta t$ is equal to zero between two elements (third term of Eq. (A.5) equal to zero), this product is constant on the whole domain, as $\boldsymbol{\Delta} t$ cannot be perpendicular to $\varphi_{0, \alpha}$ by definition (see [34]). So the solution of the problem would be $\boldsymbol{u}_{, \alpha} \cdot \boldsymbol{t}_{0}=0$ on the whole domain as $\overline{\boldsymbol{\Delta}} \boldsymbol{t}=0$ on $\partial_{T} \mathcal{A}_{h}$. Since the fourth and fifth terms of Eq. (A.5) are equal to zero, the displacement $\boldsymbol{u}$ is continuous on $\mathcal{A}_{h}$. As the constrained displacement $\overline{\boldsymbol{u}}=0$, the only remaining solution is $\boldsymbol{u}=0$ on $\mathcal{A}_{h}$.

\footnotetext{
${ }^{11}$ Except on the domain boundary $\partial_{T} \mathcal{A}_{h}$, which is neglected herein for simplicity.
} 
With the aim of demonstrating the stability of the method an upper and a lower bound of the bi-linear form (61) are given. These bounds can be obtained as in [34] with the addition of the supplementary interface terms related to the full DG formulation. After some developments similar to [30, 34] the following upper bound of the bi-linear form can be obtained,

$$
|a(\boldsymbol{u}, \delta \boldsymbol{u})|^{2} \leq C^{k}\left(\beta_{\alpha}\right)|\|\boldsymbol{u}\||^{2}|\|\delta \boldsymbol{u}\||^{2} \quad \forall \boldsymbol{u}, \delta \boldsymbol{u} \in \mathrm{U}_{c}^{\mathrm{f}}\left(\mathcal{A}_{h}\right),
$$

where $C^{k}\left(\beta_{\alpha}\right)$ is a number larger than $\max \left(4,\left(C_{\alpha}^{\prime k}\right)^{2} / \beta_{\alpha}\right)$, where $C_{\alpha}^{\prime k}>0$ are constants depending only on the degree of the polynomial approximation $k$.

Moreover, a lower bound of the bi-linear form can be written as,

$$
\begin{aligned}
& a(\boldsymbol{u}, \boldsymbol{u}) \geq\left(1-\varepsilon_{n}\right) \sum_{e}\left\|{\sqrt{\mathcal{H}_{n} \bar{j}_{0}}}^{\alpha \beta} \frac{1}{2}\left(\boldsymbol{\varphi}_{0, \alpha} \cdot \boldsymbol{u}_{, \beta}+\boldsymbol{u}_{, \alpha} \cdot \boldsymbol{\varphi}_{0, \beta}\right)\right\|_{L^{2}\left(\mathcal{A}_{e}\right)}^{2}+ \\
& \left(1-\varepsilon_{m}\right) \sum_{e}\left\|{\sqrt{\mathcal{H}_{m} \bar{j}_{0}}}^{\alpha \beta}\left(\boldsymbol{\varphi}_{0, \alpha} \cdot \boldsymbol{\Delta} \boldsymbol{t}_{, \beta}+\boldsymbol{u}_{, \alpha} \cdot \boldsymbol{t}_{0, \beta}\right)\right\|_{L^{2}\left(\mathcal{A}_{e}\right)}^{2}+ \\
& \left(1-2 \frac{C_{n}^{k^{2}}}{\varepsilon_{n} \beta_{2}}\right) \sum_{e}\left\|{\sqrt{\frac{\beta_{2} \mathcal{H}_{n} \bar{j}_{0}}{2 h^{s}}}}^{\gamma \delta} \boldsymbol{\varphi}_{0, \gamma} \cdot \llbracket \boldsymbol{u} \rrbracket \nu_{\delta}^{-}\right\|_{\mathrm{L}^{2}\left(\partial \mathcal{A}_{e}\right)}^{2}+ \\
& \left(1-2 \frac{C_{m}^{k^{2}}}{\varepsilon_{m} \beta_{1}}\right) \sum_{e}\left\|\sqrt{\frac{\beta_{1}^{\mathcal{H}_{m} \bar{j}_{0}}}{2 h^{s}}} \gamma \delta \varphi_{0, \gamma} \cdot \llbracket \boldsymbol{\Delta} \boldsymbol{t}(\boldsymbol{u}) \rrbracket \nu_{\delta}^{-}\right\|_{L^{2}\left(\partial \mathcal{A}_{e}\right)}^{2}+ \\
& \sum_{e}\left\|\sqrt{\frac{\beta_{3} \mathcal{H}_{s} \bar{j}_{0}}{2 h^{s}}} \boldsymbol{t}_{0} \cdot \llbracket \boldsymbol{u} \rrbracket \nu_{\gamma}^{-}\right\|_{\mathrm{L}^{2}\left(\partial \mathcal{A}_{e}\right)}^{2} \quad \forall \boldsymbol{u} \in \mathrm{U}_{c}^{\mathrm{f}}\left(\mathcal{A}_{h}\right) .
\end{aligned}
$$

where $\epsilon_{n}$ and $\epsilon_{m}$ are constants larger than zero coming from the so-called $\epsilon$ inequality $\forall \epsilon>0:|a b| \leq \frac{\epsilon}{2} a^{2}+\frac{1}{2 \epsilon} b^{2}$ or $\forall \epsilon>0:|a b| \leq \epsilon a^{2}+\frac{1}{4 \epsilon} b^{2}$.

The stability of the method can be proved directly from this last relation. Indeed the comparison of the right hand terms of (A.5) and (A.9) leads to,

$$
a\left(\boldsymbol{\varphi}_{h}, \boldsymbol{\varphi}_{h}\right) \geq C\left(\beta_{\alpha}\right)\left|\left\|\boldsymbol{u}_{h}\right\|\right|
$$

where $C\left(\beta_{\alpha}\right)>0$ as for given $0<\epsilon_{n}<1$ and $0<\epsilon_{m}<1$ there always exist $\beta_{1}>\frac{2\left(C_{n}^{k}\right)^{2}}{\epsilon_{n}}$ and $\beta_{2}>\frac{2\left(C_{m}^{k}\right)^{2}}{\epsilon_{m}}$. This shows that the stability of the method is conditioned by the constant $\beta_{1}$ and $\beta_{2}$ which must be large enough. Note that the equation (A.10) does not imply stability conditions on the parameter $\beta_{3}$ as long as $\beta_{3}>0$.

\section{Convergence rate in the energy norm}

The convergence rate in the energy norm can be demonstrated in the same way as in [34] and the results are reported herein only to give a complete view of numerical properties. In the following, the error between the FE solution and the polynomial interpolation of the exact solution is calculated to establish the 
convergence rate in the energy norm of the method. First some definitions and hypotheses are given. If $\boldsymbol{u} \in \mathrm{U}_{c}^{\mathrm{f}}\left(\mathcal{A}_{h}\right)$ is the exact solution of the problem, its interpolant $\boldsymbol{u}^{k}$ in the manifold $\mathrm{U}_{\mathrm{h}_{c}}^{k}$ is defined by

$$
\int_{\mathcal{A}_{h}}\left(\boldsymbol{u}-\boldsymbol{u}^{k}\right) \cdot \delta \boldsymbol{u} \bar{j}_{0} d \mathcal{A}=0 \quad \forall \delta \boldsymbol{u} \in \mathrm{U}_{\mathrm{h} c}^{k} .
$$

The error is defined as

$$
\boldsymbol{e}=\boldsymbol{u}_{h}-\boldsymbol{u} \in \mathrm{U}_{c}^{\mathrm{f}}\left(\mathcal{A}_{h}\right),
$$

where the imposed displacement on $\partial_{U} \mathcal{A}$ is strictly enforced and equal to zero, whereas the error on the exact solution interpolant is defined as

$$
\boldsymbol{e}^{k}=\boldsymbol{u}_{h}-\boldsymbol{u}^{k} \in \mathrm{U}_{\mathrm{h} c}^{k} \subset \mathrm{U}_{c}^{\mathrm{f}}\left(\mathcal{A}_{h}\right) .
$$

After some developments similar to the ones in $[30,34]$ it can be found that,

$$
\left|\left\|\boldsymbol{e}^{k}\right\|\right| \leq C\left(\beta_{1}, \beta_{2}, \beta_{3}\right) \sum_{e} h^{s-1}|\boldsymbol{u}|_{\mathbf{H}^{k+1}\left(l_{e}\right)} .
$$

The order of convergence is one order lower than the degree of polynomial approximation, which is consistent with the presence of high-order derivatives in the governing equations (3).

\section{Convergence in the $\mathbf{L}^{2}$ norm}

The convergence of the solution in the $\mathbf{L}^{2}$ norm is demonstrated under the two assumptions:

1. Proper elliptic regularity of the problem

2. Pure Dirichlet boundary conditions (i.e. $\overline{\boldsymbol{u}}=\overline{\boldsymbol{t}}=0$ on $\partial \mathcal{A}_{h}$ )

As well as the convergence rate in the energy norm, the demonstration of the convergence in the $\mathbf{L}^{2}$ norm follows the method presented in [34], so only the final result is given here,

$$
\|\boldsymbol{e}\|_{\mathbf{L}^{2}\left(l_{e}\right)} \leq\left\{\begin{array}{l}
\sum_{e} C h^{s k+1}|\boldsymbol{u}|_{\mathbf{H}^{k+1}\left(l_{e}\right)} \text { if } k>2 \\
\sum_{e} C h^{s 2}|\boldsymbol{u}|_{\mathbf{H}^{3}\left(l_{e}\right)} \text { if } k=2
\end{array},\right.
$$

where the case $k=2$ is obtained by following the work of Wells et al. [33]. The relation (A.15) demonstrates that the method has an optimal convergence rate for at least cubic elements. 


\section{References}

[1] D. S. Dugdale, Yielding of steel sheets containing slits, Journal of the Mechanics and Physics of Solids 8 (2) (1960) 100-104, ISSN 0022-5096.

[2] G. Barenblatt, The Mathematical Theory of Equilibrium Cracks in Brittle Fracture, vol. Volume 7, Elsevier, 55-129, 1962.

[3] G. T. Camacho, M. Ortiz, Computational modelling of impact damage in brittle materials, International Journal of Solids and Structures 33 (20-22) (1996) 2899-2938, ISSN 0020-7683.

[4] M. Anvari, J. Liu, C. Thaulow, Dynamic ductile fracture in aluminum round bars: experiments and simulations, International Journal of Fracture 143 (4) (2007) 317-332-, URL http://dx.doi.org/10.1007/s10704-007-9062-9.

[5] P. M. A. Areias, J. H. Song, T. Belytschko, Analysis of fracture in thin shells by overlapping paired elements, Computer Methods in Applied Mechanics and Engineering 195 (41-43) (2006) 5343-5360, ISSN 0045-7825.

[6] F. Cirak, M. Ortiz, A. Pandolfi, A cohesive approach to thin-shell fracture and fragmentation, Computer Methods in Applied Mechanics and Engineering 194 (21-24) (2005) 2604-2618, ISSN 0045-7825.

[7] J. F. Molinari, G. Gazonas, R. Raghupathy, A. Rusinek, F. Zhou, The cohesive element approach to dynamic fragmentation: the question of energy convergence, International Journal for Numerical Methods in Engineering 69 (3) (2007) 484-503, ISSN 1097-0207, URL http://dx.doi.org/10.1002/nme.1777.

[8] A. Pandolfi, P. Krysl, M. Ortiz, Finite element simulation of ring expansion and fragmentation: The capturing of length and time scales through cohesive models of fracture, International Journal of Fracture 95 (1) (1999) 279-297, URL http://dx.doi.org/10.1023/A:1018672922734.

[9] I. Scheider, W. Brocks, Simulation of cup-cone fracture using the cohesive model, Engineering Fracture Mechanics 70 (14) (2003) 1943-1961, ISSN 0013-7944.

[10] R. R. Settgast, M. M. Rashid, Continuum coupled cohesive zone elements for analysis of fracture in solid bodies, Engineering Fracture Mechanics 76 (11) (2009) 1614 - 1635, ISSN 0013-7944, doi:DOI: 10.1016/j.engfracmech.2009.02.024.

[11] A. Seagraves, R. Radovitzky, Advances in Cohesive Zone Modeling of Dynamic Fracture, in: A. Shukla, G. Ravichandran, Y. D. Rajapakse (Eds.), Dynamic Failure of Materials and Structures, Springer US, ISBN 978-1-4419-0446-1, 349-405, URL http://dx.doi.org/10.1007/978-1-4419-0446-1_12, 2010. 
[12] R. Radovitzky, A. Seagraves, M. Tupek, L. Noels, A scalable 3D fracture and fragmentation algorithm based on a hybrid, discontinuous Galerkin, Cohesive Element Method, Computer Methods in Applied Mechanics and Engineering 200 (2011) 326-344.

[13] V. Tvergaard, Crack growth predictions by cohesive zone model for ductile fracture, Journal of the Mechanics and Physics of Solids 49 (9) (2001) 2191-2207, ISSN 0022-5096.

[14] P. A. Klein, J. W. Foulk, E. P. Chen, S. A. Wimmer, H. J. Gao, Physicsbased modeling of brittle fracture: cohesive formulations and the application of meshfree methods, Theoretical and Applied Fracture Mechanics 37 (1-3) (2001) 99-166, ISSN 0167-8442.

[15] W. Celes, A compact adjacencybased topological data structure for finite element mesh representation, International journal for numerical methods in engineering 64 (11) (2005) 1529-1556, ISSN 0029-5981.

[16] Z. J. Zhang, G. H. Paulino, W. Celes, Extrinsic cohesive modelling of dynamic fracture and microbranching instability in brittle materials, International Journal for Numerical Methods in Engineering 72 (8) (2007) 893-923, URL http://dx.doi.org/10.1002/nme.2030.

[17] A. Mota, J. Knap, M. Ortiz, Fracture and fragmentation of simplicial finite element meshes using graphs, International Journal for Numerical Methods in Engineering 73 (11) (2008) 1547-1570, URL http://dx.doi.org/10.1002/nme.2135.

[18] I. Dooley, S. Mangala, L. Kale, P. Geubelle, Parallel Simulations of Dynamic Fracture Using Extrinsic Cohesive Elements, J. Sci. Comput. 39 (1) (2009) 144-165.

[19] F. Perales, S. Bourgeois, A. Chrysochoos, Y. Monerie, Two field multibody method for periodic homogenization in fracture mechanics of nonlinear heterogeneous materials, Engineering Fracture Mechanics 75 (11) (2008) 33783398, ISSN 0013-7944.

[20] V. Acary, Y. Monerie, V. Acary, Y. Monerie, A. V. et al, Nonsmooth fracture dynamics using a cohesive zone approach, Tech. Rep., INRIA, URL http://hal.inria.fr/inria-00110560/en/, 2006.

[21] R. Abedi, M. A. Hawker, R. B. Haber, K. Matouscaron, An adaptive spacetime discontinuous Galerkin method for cohesive models of elastodynamic fracture, International Journal for Numerical Methods in Engineering 81 (10) (2010) 1207-1241, URL http://dx.doi.org/10.1002/nme.2723.

[22] J. Mergheim, E. Kuhl, P. Steinmann, A hybrid discontinuous Galerkin/interface method for the computational modelling of failure, Communications in Numerical Methods in Engineering 20 (7) (2004) 511-519, URL http://dx.doi.org/10.1002/cnm.689. 
[23] M. Prechtel, P. Ronda, R. Janisch, A. Hartmaier, G. Leugering, P. Steinmann, M. Stingl, Simulation of fracture in heterogeneous elastic materials with cohesive zone models, International Journal of Fracture 168 (2011) 15-29, ISSN 0376-9429, URL http://dx.doi.org/10.1007/s10704-010-9552-z, 10.1007/s10704-0109552-z.

[24] R. Larsson, J. Mediavilla, M. Fagerstrom, Dynamic fracture modeling in shell structures based on XFEM, Int. J. Numer. Meth. Engng. (2010) n/an/aISSN 1097-0207, URL http://dx.doi.org/10.1002/nme.3086.

[25] N. Moës, J. Dolbow, T. Belytschko, A finite element method for crack growth without remeshing, International Journal for Numerical Methods in Engineering 46 (1) (1999) 131-150, ISSN 1097-0207.

[26] N. Moës, T. Belytschko, Extended finite element method for cohesive crack growth, Engineering Fracture Mechanics 69 (7) (2002) 813-833, ISSN 00137944.

[27] E. Bchet, H. Minnebo, N. Moës, B. Burgardt, Improved implementation and robustness study of the X-FEM for stress analysis around cracks, International Journal for Numerical Methods in Engineering 64 (8) (2005) 1033-1056, ISSN 1097-0207, doi:10.1002/nme.1386, URL http://dx.doi.org/10.1002/nme.1386.

[28] S. Mohammadi, Frontmatter, Blackwell Publishing Ltd, ISBN 9780470697795, i-xvii, doi:10.1002/9780470697795.fmatter, URL http://dx.doi.org/10.1002/9780470697795.fmatter, 2008.

[29] M. Duflot, E. Wyart, F. Lani, P. Martiny, S. Sagnier, Application of XFEM to multi-site crack propagation, Engineering Fracture Mechanics submitted.

[30] G. Becker, L. Noels, A fracture framework for Euler-Bernoulli beams based on a full discontinuous Galerkin formulation/extrinsic cohesive law combination, International Journal for Numerical Methods in Engineering 85 (10) (2011) 1227-1251, ISSN 1097-0207, doi:10.1002/nme.3008, URL http://dx.doi.org/10.1002/nme.3008.

[31] G. Engel, K. Garikipati, T. J. R. Hughes, M. G. Larson, L. Mazzei, R. L. Taylor, Continuous/discontinuous finite element approximations of fourthorder elliptic problems in structural and continuum mechanics with applications to thin beams and plates, and strain gradient elasticity, Computer Methods in Applied Mechanics and Engineering 191 (34) (2002) 3669-3750, ISSN 0045-7825.

[32] P. Hansbo, M. G. Larson, A discontinuous Galerkin method for the plate equation, Calcolo 39 (1) (2002) 41-59, URL http://dx.doi.org/10.1007/s100920200001. 
[33] G. N. Wells, N. T. Dung, A C0 discontinuous Galerkin formulation for Kirchhoff plates, Computer Methods in Applied Mechanics and Engineering 196 (35-36) (2007) 3370-3380, ISSN 0045-7825.

[34] L. Noels, R. Radovitzky, A new discontinuous Galerkin method for Kirchhoff-Love shells, Computer Methods in Applied Mechanics and Engineering 197 (33-40) (2008) 2901-2929, ISSN 0045-7825.

[35] L. Noels, A discontinuous Galerkin formulation of non-linear Kirchhoff-Love shells, International Journal for Numerical Methods in Engineering 78 (3) (2009) 296-323, ISSN 1097-0207, URL http://dx.doi.org/10.1002/nme.2489.

[36] N. Dung, G. Wells, Geometrically nonlinear formulation for thin shells without rotation degrees of freedom, Computer Methods in Applied Mechanics and Engineering 197 (2008) 2778-2788, URL http://dx.doi.org/10.1016/j.cma.2008.01.001.

[37] J. C. Simo, D. D. Fox, M. S. Rifai, On a stress resultant geometrically exact shell model. Part II: the linear theory; computational aspects, Comput. Methods Appl. Mech. Eng. 73 (1) (1989) 53-92.

[38] J. C. Simo, D. D. Fox, On stress resultant geometrically exact shell model. Part I: formulation and optimal parametrization, Comput. Methods Appl. Mech. Eng. 72 (3) (1989) 267-304.

[39] L. Noels, R. Radovitzky, An explicit discontinuous Galerkin method for non-linear solid dynamics: Formulation, parallel implementation and scalability properties, International Journal for Numerical Methods in Engineering 74 (9) (2008) 1393-1420, ISSN 1097-0207, URL http://dx.doi.org/10.1002/nme.2213.

[40] D. N. Arnold, F. Brezzi, B. Cockburn, L. D. Marini, Unified Analysis of Discontinuous Galerkin Methods for Elliptic Problems, SIAM Journal on Numerical Analysis 39 (5) (2002) 1749-1779, ISSN 00361429, URL http://www. jstor.org/stable/4101034.

[41] A. Lew, P. Neff, D. Sulsky, M. Ortiz, Optimal BV estimates for a discontinuous Galerkin method for linear elasticity, Appl Math Res Express 2004 (3) (2004) 73-106, URL http://amrx.oxfordjournals.org/cgi/content/abstract/2004/3/73.

[42] L. Noels, R. Radovitzky, A general discontinuous Galerkin method for finite hyperelasticity. Formulation and numerical applications, International Journal for Numerical Methods in Engineering 68 (1) (2006) 64-97, ISSN 1097-0207, URL http://dx.doi.org/10.1002/nme.1699.

[43] A. T. Eyck, A. Lew, Discontinuous Galerkin methods for nonlinear elasticity, International Journal for Numerical Methods 
in Engineering 67 (9) (2006) 1204-1243, ISSN 1097-0207, URL http://dx.doi.org/10.1002/nme.1667.

[44] L. Noels, R. Radovitzky, Alternative Approaches for the Derivation of Discontinuous Galerkin Methods for Nonlinear Mechanics, J. Appl. Mech. 74 (5) (2007) 1031-1036, doi:http://dx.doi.org/10.1115/1.2712228, URL http://link.aip.org/link/?AMJ/74/1031/1.

[45] A. Lew, A. Eyck, R. Rangarajan, Some Applications of Discontinuous Galerkin Methods in Solid Mechanics, IUTAM Symposium on Theoretical, Computational and Modelling Aspects of Inelastic Media (2008) 227-236.

[46] M. Ortiz, A. Pandolfi, Finite-Deformation Irreversible Cohesive Elements for Three-Dimensional Crack Propagation Analysis, International Journal for Numerical Methods in Engineering 44 (2000) 44-1267.

[47] A. Corigliano, F. Cacchione, A. Frangi, S. Zerbini, Numerical modelling of impact rupture in polysilicon microsystems, Computational Mechanics 42 (2008) 251-259, ISSN 0178-7675, URL http://dx.doi.org/10.1007/s00466-007-0231-5, 10.1007/s00466-0070231-5.

[48] H. Li, N. Chandra, Analysis of crack growth and crack-tip plasticity in ductile materials using cohesive zone models, International Journal of Plasticity 19 (6) (2003) 849-882-, ISSN 0749-6419.

[49] A. Pandolfi, M. Ortiz, An Efficient Adaptive Procedure for Three-Dimensional Fragmentation Simulations, Engineering with Computers 18 (2) (2002) 148-159, URL http://dx.doi.org/10.1007/s003660200013.

[50] K. D. Papoulia, C.-H. Sam, S. A. Vavasis, Time continuity in cohesive finite element modeling, International Journal for $\mathrm{Nu}-$ merical Methods in Engineering 58 (5) (2003) 679-701, URL http://dx.doi.org/10.1002/nme.778.

[51] P. Zavattieri, Modeling of crack propagation in thin-walled structures, Mecanica Computacional XXIII (2004) 209-228.

[52] C. Geuzaine, J.-F. Remacle, Gmsh: A 3-D finite element mesh generator with built-in pre- and post-processing facilities, International Journal for Numerical Methods in Engineering 79 (11) (2009) 1309-1331, URL http://dx.doi.org/10.1002/nme.2579.

[53] S. Toledo, D. Chen, V. Rotkin, TAUCS Web page, http://www.tau.ac.il/ stoledo/taucs/, 2003.

[54] S. Toledo, D. Chen, V. Rotkin, TAUCS: A Library of Sparse Linear Solvers, Tech. Rep. version 2.2, Tel-Aviv University, 2003. 
[55] S. Balay, J. Brown, K. Buschelman, W. D. Gropp, D. Kaushik, M. G. Knepley, L. C. McInnes, B. F. Smith, H. Zhang, PETSc Web page, http://www.mcs.anl.gov/petsc, 2011.

[56] S. Balay, J. Brown, , K. Buschelman, V. Eijkhout, W. D. Gropp, D. Kaushik, M. G. Knepley, L. C. McInnes, B. F. Smith, H. Zhang, PETSc Users Manual, Tech. Rep. ANL-95/11 - Revision 3.1, Argonne National Laboratory, 2010.

[57] S. Balay, W. D. Gropp, L. C. McInnes, B. F. Smith, Efficient Management of Parallelism in Object Oriented Numerical Software Libraries, in: E. Arge, A. M. Bruaset, H. P. Langtangen (Eds.), Modern Software Tools in Scientific Computing, Birkhäuser Press, 163-202, 1997.

[58] G. M. Hulbert, J. Chung, Explicit time integration algorithms for structural dynamics with optimal numerical dissipation, Computer Methods in Applied Mechanics and Engineering 137 (2) (1996) 175 188, ISSN 0045-7825, doi:DOI: 10.1016/S0045-7825(96)01036-5, URL http://www. sciencedirect. com/science/article/B6V29-3WFNRDY-5/ 2/2ec79b924e3dd1b9231ffe40c6a40306.

[59] P. D. Zavattieri, Modeling of Crack Propagation in Thin-Walled Structures Using a Cohesive Model for Shell Elements, J. Appl. Mech. 73 (6) (2006) 948-958, URL http://link. aip.org/link/?AMJ/73/948/1.

[60] F. Zhou, J.-F. Molinari, Stochastic fracture of ceramics under dynamic tensile loading, International Journal of Solids and Structures 41 (22-23) (2004) 6573 - 6596, ISSN 0020-7683, doi:DOI: 10.1016/j.ijsolstr.2004.05.029, URL http://www. sciencedirect.com/science/article/pii/S0020768304002562. 


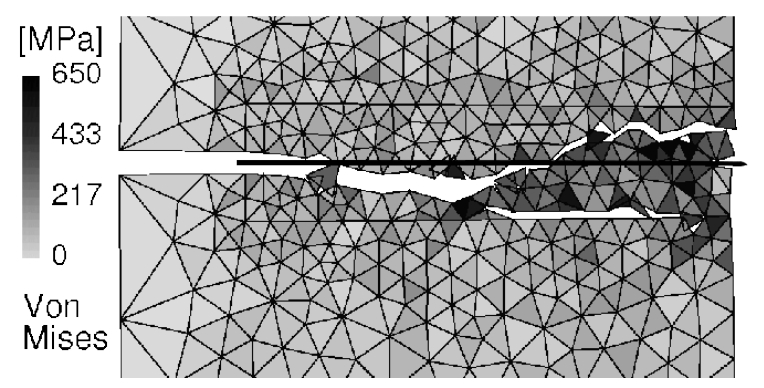

(a)

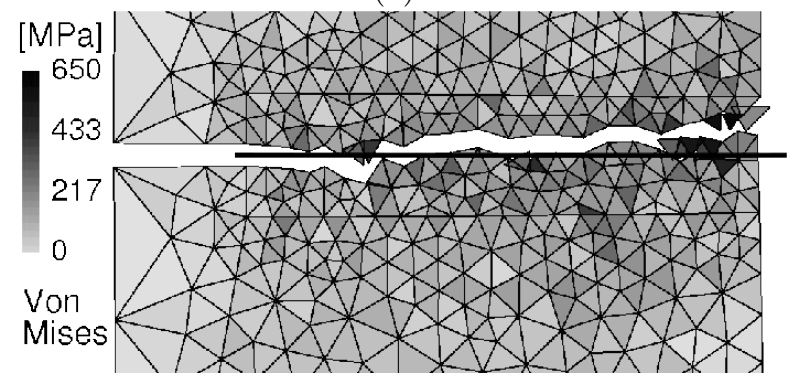

(b)

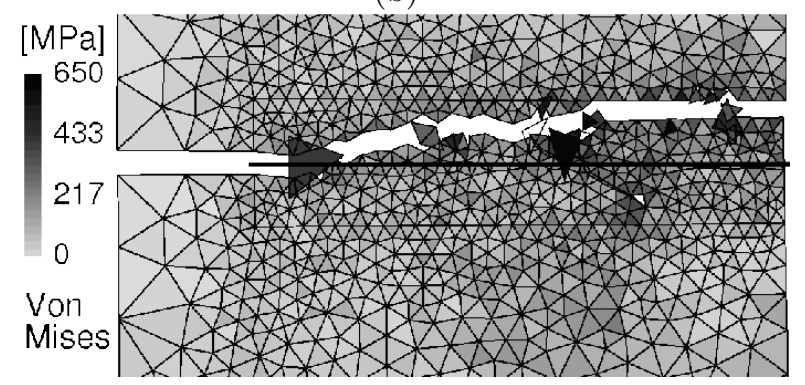

(c)

Figure 13: Crack path obtained with unstructured meshes for tensile benchmark. a) Coarse mesh without dissipation. b) Coarse mesh with numerical damping. c) Fine mesh with numerical damping. The black line draws the "exact" crack path. The displacements are magnified 10 times. 


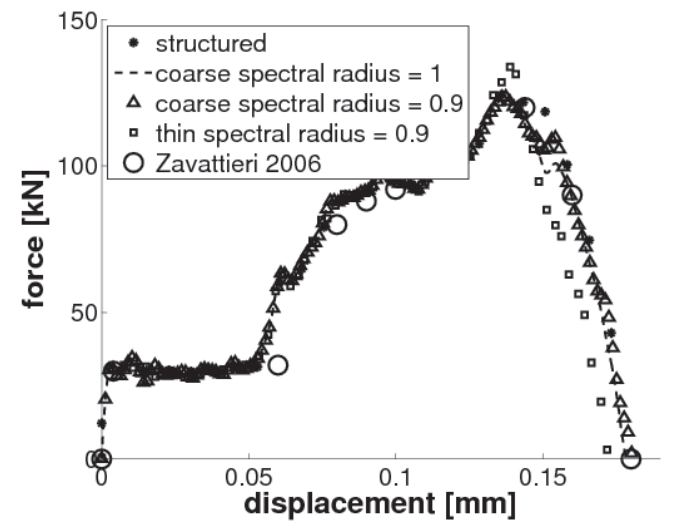

Figure 14: Force-displacement relation obtained with different meshes for the tensile test.

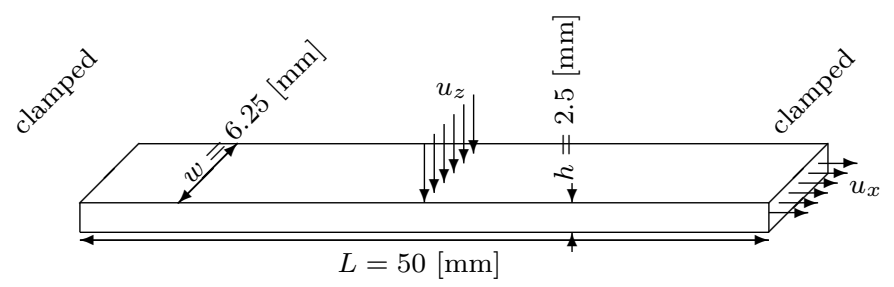

Figure 15: Configuration of the double clamped plate. 


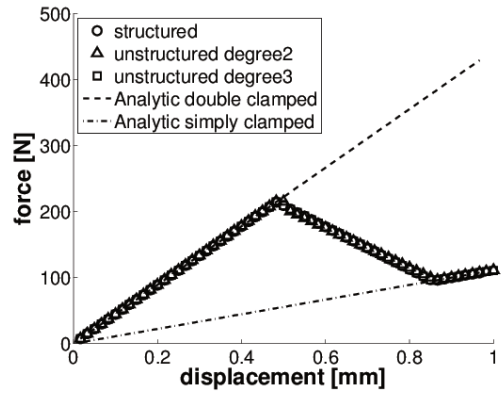

(a)

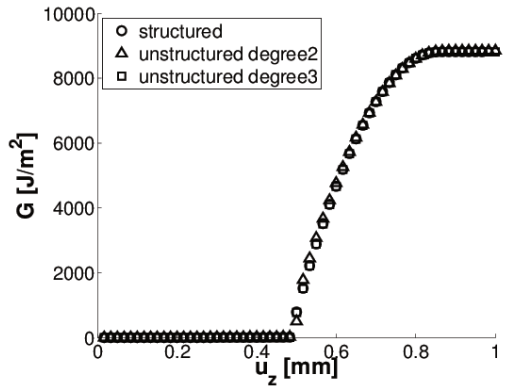

(b)

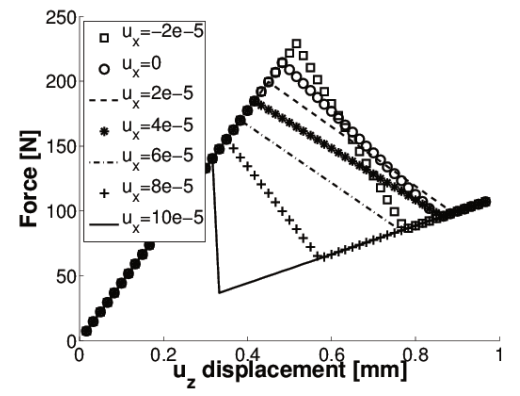

(c)

Figure 16: Results of double clamped plate. (a) Force vs. displacement curve without pretension. (b) Released energy without pretension. (c) Force vs. displacement curve for different values of pretension.

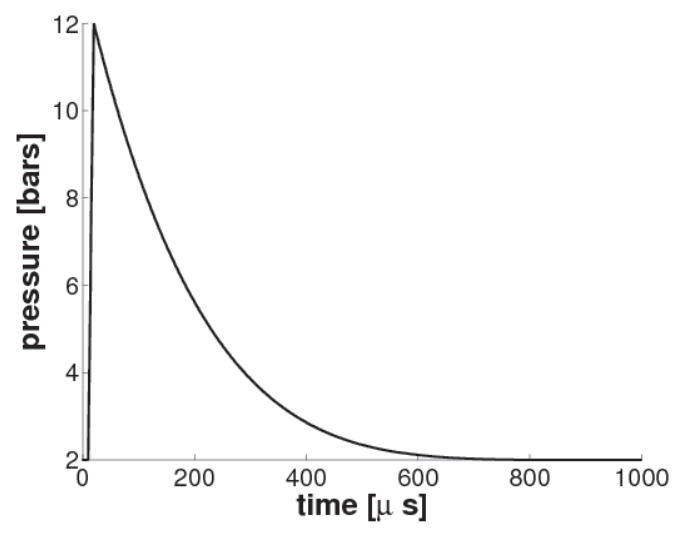

Figure 17: Applied pressure for the cylinder test. 


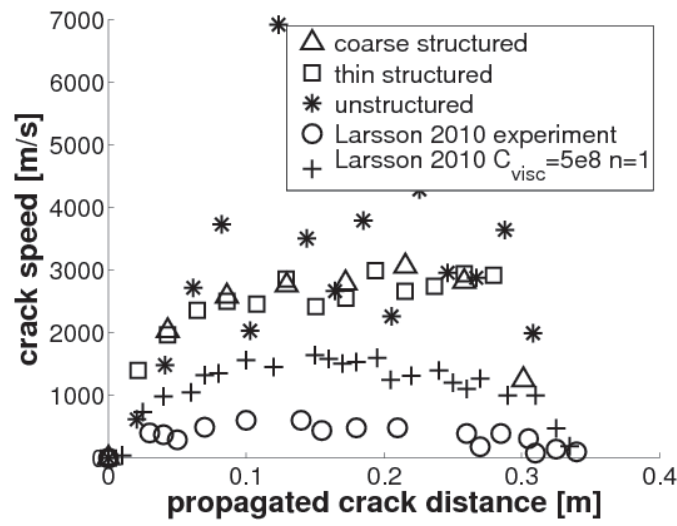

Figure 18: Evolution of crack speed with respect of crack propagation 

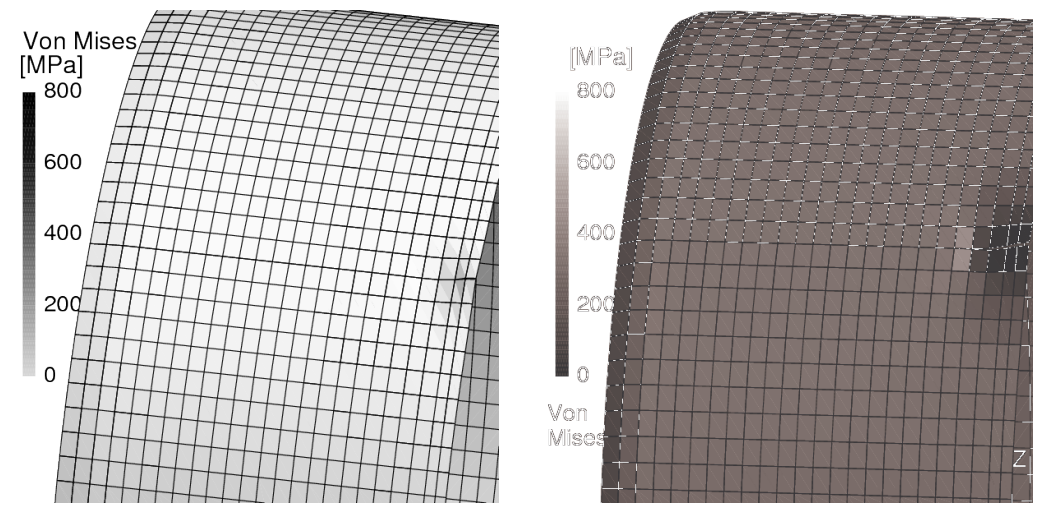

(a) $0[\mu s]$
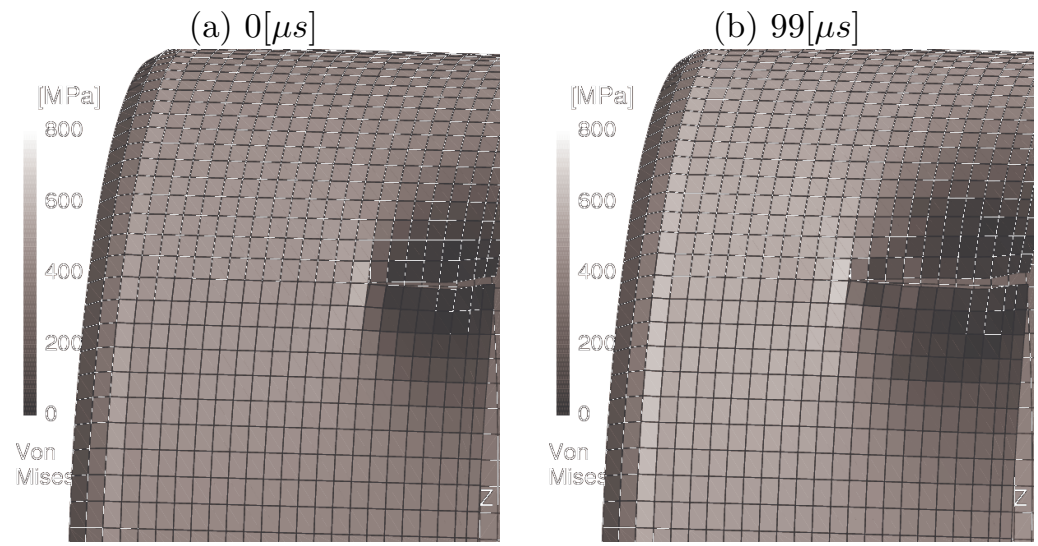

(c) $140[\mu s]$

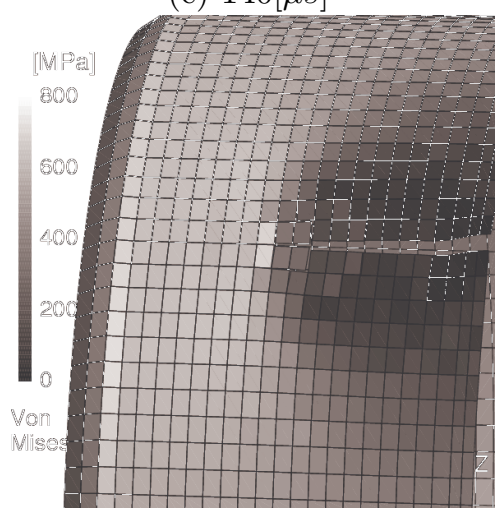

(d) $161[\mu s]$

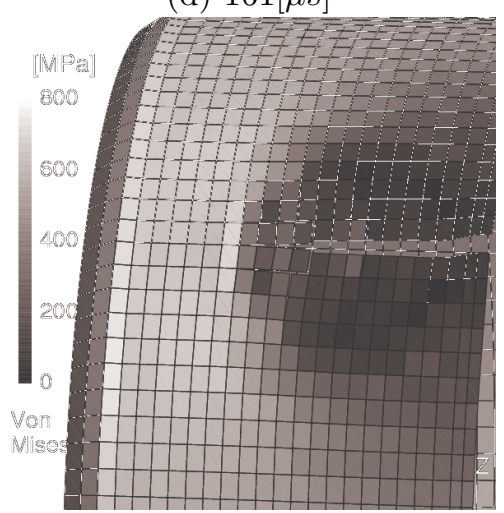

(e) $180[\mu s]$

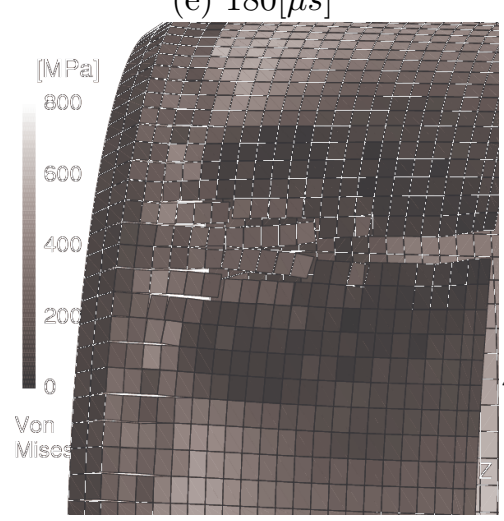

(f) $189[\mu s]$

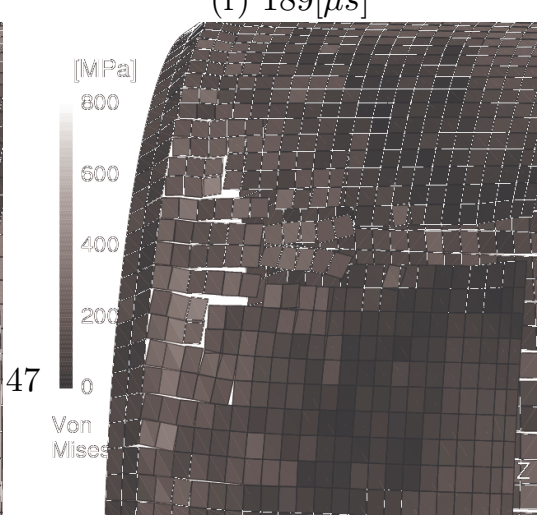

(g) $226[\mu s]$

(h) $266[\mu s]$

Figure 19: Crack propagation of the cylinder test with the finest structured mesh. Displacements are magnified 5 times. 


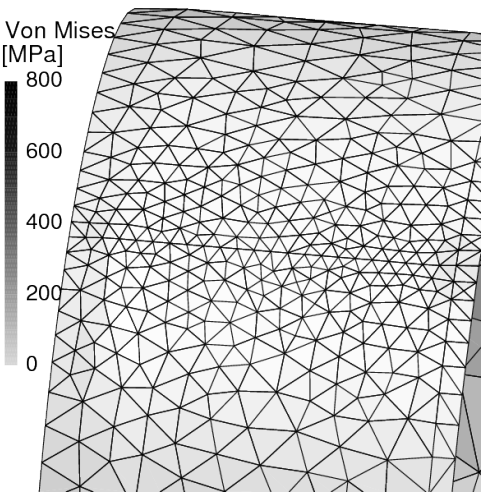

(a) $0[\mu s]$

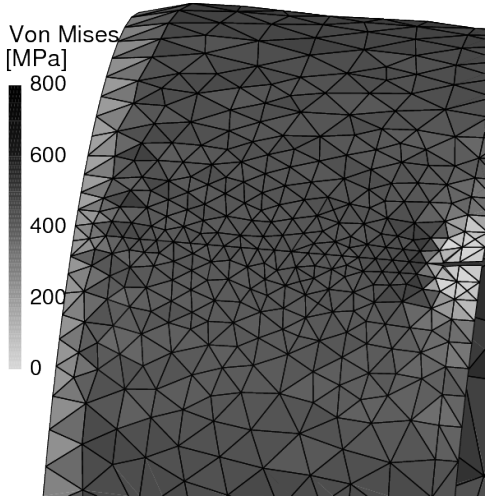

(c) $140[\mu s]$

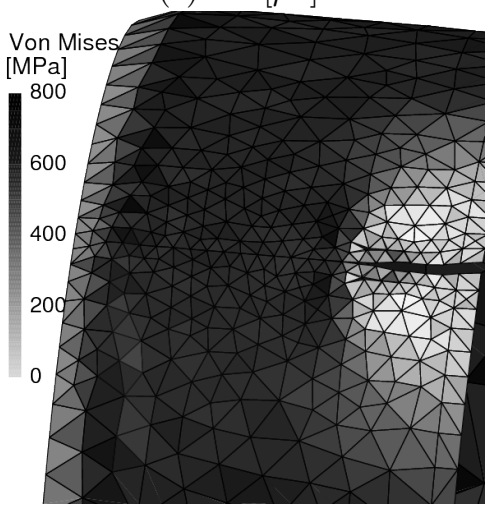

(e) $180[\mu \mathrm{s}]$

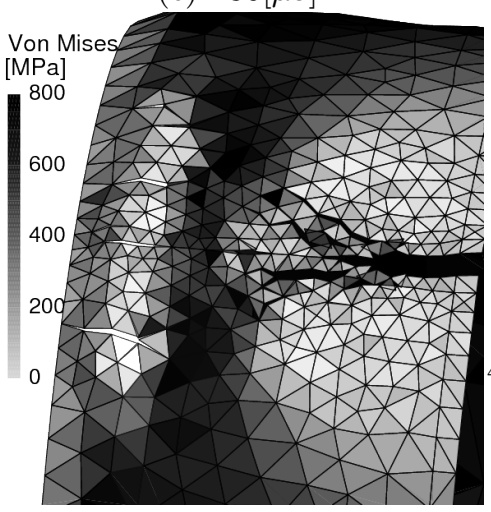

(g) $226[\mu s]$

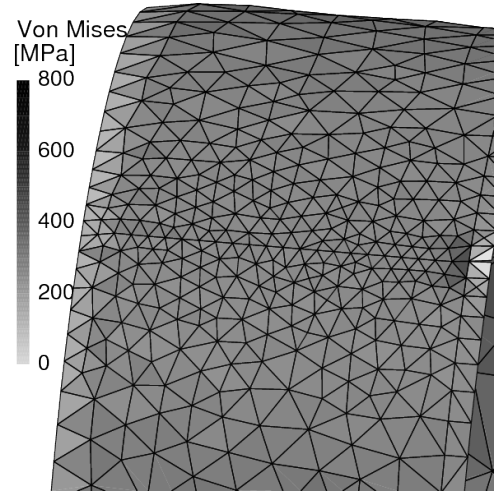

(b) $99[\mu s]$

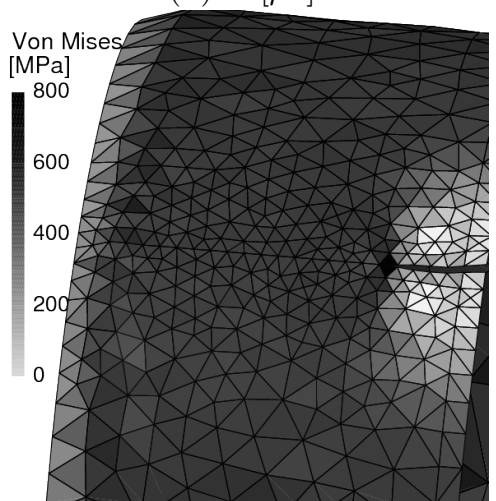

(d) $161[\mu s]$

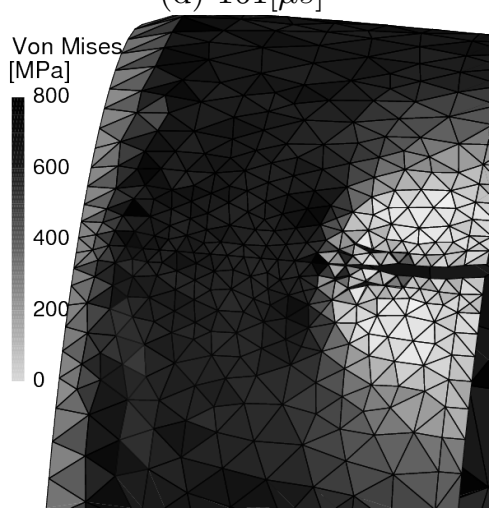

(f) $189[\mu s]$

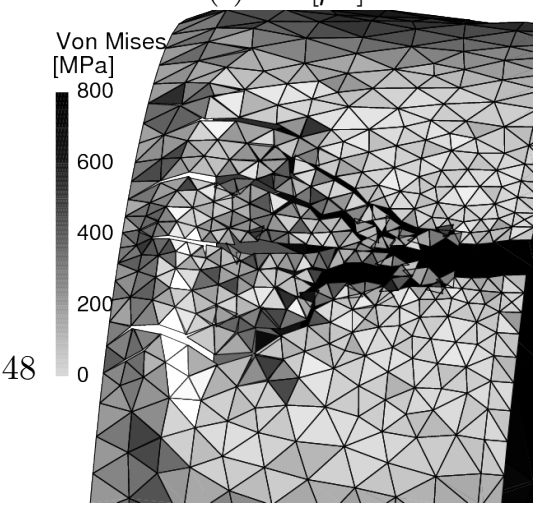

(h) $266[\mu s]$

Figure 20: Crack propagation of the cylinder test with an unstructured mesh. Displacements are magnified 5 times. 